\title{
LARGE DEVIATION PRINCIPLE FOR MODERATE DEVIATION PROBABILITIES OF BOOTSTRAP EMPIRICAL MEASURES
}

\author{
M. S. ERMAKOV
}

\section{INTRODUCTION}

Large deviation principle (LDP) for i.i.d.r.v.'s ( 2, 4, 8, 10, 13, 15, 17, 24]) allows to study a large number of different problems on large deviation probabilities of statistics. LDP for the bootstrap empirical measures has been studied not in such a large number of papers ( [ $[\underline{6}$ and [7]). The goal of the paper is to prove LDP for the conditional distribution of bootstrap empirical measure given empirical measure and similar LDP for the common distribution of bootstrap empirical measure and empirical measure. To simplify the terminology these LDP will be called moderate deviation principles (MDP) (see [2]). MDP for the conditional distribution of bootstrap empirical measure given empirical measure will be called the conditional principle of moderate deviation probabilities.

For bootstrap sample means the conditional LDP has been established in 22 . For bootstrap empirical measures such a version of LDP has been proved in [6]. The strong asymptotics of moderate deviation probabilities of bootstrap sample means have been studied in [9] and [28.

The interest to the problem under consideration is caused the following reasons.

The conditional MDP for bootstrap empirical measures holds for significantly wider zones of moderate deviation probabbilities than MDP for empirical measures. Thus the normal approximation for differentiable statistical functionals depending on bootstrap empirical measures works for significantly wider zones than the normal approximation for the functionals depending on empirical measures. At the same time MDP for the common distributions of bootstrap empirical measures and empirical measures holds for a much narrower zone of moderate deviation probabilities than MDP for empirical probability measures. This result shows significant instability of bootstrap if the empirical probability measure lies in the moderate deviation zone.

As have been shown in [15] and [16, MDP for empirical measures and technique of differentiation in functional spaces allow to establish MDP for differentiable statistical functionals. It turns out that this technique works to the same extent as in the proof of asymptotic normality [27. The paper allows to obtain similar results for differentiable functionals depending on the bootstrap empirical measures.

Suppose that

$-S$ is Hausdorff topological space;

- $\mathcal{F}$ is $\sigma$-field of Borel sets on $S$;

$-\Lambda$ is the set of all probability measures on $(S, \mathcal{F})$.

Let $X_{1}, \ldots, X_{n}$ be independent identically distributed random variables having probability measure $\mathbf{P} \in \Lambda$.

Denote $\widehat{\mathbf{P}}_{n}$ empirical measure of $X_{1}, \ldots, X_{n}$.

Date: 12 november 2012.

Key words and phrases. large deviation principle, moderate deviations, bootstrap, empirical measure.

Paper was supported RFFI Grant 11-01-00769-. 
In 1979, in a landmark paper Efron 12 proposed to analyze the distributions of statistics $V\left(X_{1}, \ldots, X_{n}\right)$ with the help of the bootstrap procedure. In the bootstrap we consider the empirical measure $\widehat{\mathbf{P}}_{n}$ as an estimator of the probability measure (pm) $\mathbf{P}$ and simulate the distribution of statistics $V\left(X_{1}, \ldots, X_{n}\right)$ on the base of $\operatorname{pm} \widehat{\mathbf{P}}_{n}$. In other words, we simulate independent copies $\left(X_{1 i}^{*}, \ldots, X_{n i}^{*}\right)_{i \in[1, k]}$ of i.i.d random variables such that $X_{1 i}^{*}$ is distributed according to $\widehat{\mathbf{P}}_{n}$. After that the empirical distribution of $\left(V\left(X_{1 i}^{*}, \ldots, X_{n i}^{*}\right)\right)_{i \in[1, k]}$ is postulated as an estimate of the distribution of $V\left(X_{1}, \ldots, X_{n}\right)$.

It is of interest to estimate large and moderate deviation probabilities of $V\left(X_{1}, \ldots, X_{n}\right)$. Such problems emerge constantly in confidence estimation and hypothesis testing. The significant levels in the confidence estimation and the type I error probabilities in hypothesis testing are (usually) of small values and thus are compatible with LDP - MDP analysis. Hence it appears natural to compare $V\left(X_{1}, \ldots, X_{n}\right)$ and $V\left(X_{1}^{*}, \ldots, X_{n}^{*}\right)$ in terms of LDP - MDP approach.

In this paper we carry out such an MDP based comparisons in the following setup.

We represent $V\left(X_{1}, \ldots, X_{n}\right)$ and $V\left(X_{1}^{*}, \ldots, X_{n}^{*}\right)$ as functionals of $\widehat{\mathbf{P}}_{n}$ and $\mathbf{P}_{n}^{*}$ respectively, where $\mathbf{P}_{n}^{*}$ is the empirical probability measure of $X_{1}^{*}, \ldots, X_{n}^{*}$ called the bootstrap empirical measure, i.e.

$$
\begin{aligned}
V\left(X_{1}, \ldots, X_{n}\right) & =T\left(\widehat{\mathbf{P}}_{n}\right), \\
V\left(X_{1}^{*}, \ldots, X_{n}^{*}\right) & =T\left(\mathbf{P}_{n}^{*}\right) .
\end{aligned}
$$

Thus we reduce the problem to the study of moderate deviation probabilities of $T\left(\mathbf{P}_{n}^{*}\right)-T\left(\widehat{\mathbf{P}}_{n}\right)$.

The paper is organized as follows.

Theorems 2.1 and 2.2 on conditional MDP for the bootstrap empirical measures are provided in section 2. Theorem 2.1 states that the conditional MDP holds almost surely. Theorem 2.2 explores rates of convergence in the conditional MDP. The results were established in terms of the $\tau_{\Theta}$ - topology allowing to study moderate deviation probabilities for unbounded statistical functionals.

In section $3 \mathrm{MDP}$ for the common distribution of empirical measures and bootstrap empirical measures are provided. The example given in section 3 shows that the weak $\tau_{\Phi}$-topology considered in this MDP could not be improved significantly.

In section 4 we discuss the extensions of these results on the case of differentiable statistical functionals. We show that the technique developed in [15] and [16] can be also implemented for the bootstrap setup. In particular MDP for the bootstrap empirical quantile processes and the bootstrap empirical copula functions are provided.

Probabilities of moderate deviations of statistics have been studied in numerous works (see 11, 3, 19, 20] and references therein). Last time this problem was explored in terms of differentiable statistical functionals [15, 16].

In sections 5, 6 and 7 the proofs of Theorems of sections 2 and 3 are provided.

We shall implement the following notations:

$-\mathbf{Q}<<\mathbf{P}$, if $\mathbf{Q} \in \Lambda$ is absolutely continuous with respect to $\mathbf{P} \in \Lambda$;

- $\mathbf{Q}_{2} \times \mathbf{Q}_{1}$ - the Cartesian product of probability measures $\mathbf{Q}_{2}, \mathbf{Q}_{1} \in \Lambda$;

$-\Lambda^{2}=\Lambda \times \Lambda$ denote the set of all probability measures $\mathbf{Q}_{2} \times \mathbf{Q}_{1}$ with $\mathbf{Q}_{2}, \mathbf{Q}_{1} \in \Lambda$;

$-C, c-$ positive constants;

- $\chi(A)$ - indicator of event $A$;

- $\int$ denote always $\int$. 


\section{Conditional MDP for Bootstrap empirical MEASURes}

2.1. The $\tau_{\Sigma}$-topologies. Let $\Sigma$ be a set of functions $f: S \rightarrow R^{1}$ such that $\mathbf{E}[|f(X)|]<\infty$. We suppose that $\Sigma$ contains the set of all bounded functions.

Denote

$$
\Lambda_{\Sigma}=\left\{\mathbf{P} \in \Lambda: \int|f(X)| d \mathbf{P}<\infty, \quad f \in \Sigma\right\} .
$$

Topology of weak convergence in $\Lambda_{\Sigma}$ providing the continuous mapping

$$
\mathbf{Q} \Rightarrow \int f d \mathbf{Q} \text { for all } f \in \Sigma, \mathbf{Q} \in \Lambda_{\Sigma}
$$

is known as the $\tau_{\Sigma^{-}}$-topology (henceforth, all topological concepts refer to the $\tau_{\Sigma^{-}}$ topology). Denote $\sigma_{\Sigma}$ the smallest $\sigma$-field that makes all these mapping measurable. For any set $\Omega \subset \Lambda_{\Sigma}$ the notations: $\mathfrak{c l}(\Omega)$ and $\mathfrak{i n t}(\Omega)$ are used for the closure and the interior of $\Omega$ respectively.

For the set $\Sigma=\Theta_{0}$ of all bounded measurable functions, the $\tau_{\Theta_{0}}$-topology is called the $\tau$-topology (see [10, 13, 17]). Define the set $\Lambda_{0 \Sigma}$ of all signed measures $\mathbf{G}, \mathbf{G}(S)=0$, having bounded variation and such that

$$
\int|f| d|\mathbf{G}|<\infty
$$

The measure $|\mathbf{G}|$ is defined as follows. For any set $A \in \mathcal{F},|\mathbf{G}|(A)$ is variation of set $A$ for signed measure $\mathbf{G}$.

The $\tau_{\Sigma}$-topology in $\Lambda_{0 \Sigma}$ is defined by a standard way. The definitions of $\mathfrak{c l}\left(\Omega_{0}\right)$ and $\operatorname{int}\left(\Omega_{0}\right), \Omega_{0} \subset \Lambda_{0 \Sigma}$, are also standard.

2.2. Rate function. For $\mathbf{G} \in \Lambda_{0}$, let

$$
\rho_{0}^{2}(\mathbf{G}, \mathbf{P})= \begin{cases}\frac{1}{2} \int\left(\frac{d \mathbf{G}}{d \mathbf{P}}\right)^{2} d \mathbf{P}, & \mathbf{G} \ll \mathbf{P}, \\ \infty, & \text { otherwise }\end{cases}
$$

be the rate function (in statistical terms, $2 \rho_{0}^{2}(G \mid P)$ is the Fisher information) which arises naturally in the MDP analysis of empirical measures $\hat{P}_{n}$ (see [4] [16], [2] and [15] ).

For the set $\Omega_{0} \subset \Lambda_{0 \Sigma}$ denote

$$
\rho_{0}^{2}\left(\Omega_{0}, \mathbf{P}\right)=\inf \left\{\rho_{0}^{2}(\mathbf{G}, \mathbf{P}), \mathbf{G} \in \Omega_{0}\right\} .
$$

2.3. Outer and inner probabilities. The empirical distribution function is not measurable (see [8, 16, 21]). By this reason, the results will be given in terms of outer and inner probabilities. Let $(\Upsilon, \mathcal{F}, \mathbf{P})$ be probability space. The outer probability of set $B \subset \Upsilon$ equals

$$
(\mathbf{P})^{*}(B)=\inf \{\mathbf{P}(A) ; B \subseteq A, A \in \operatorname{Im}\},
$$

and its inner probability equals $(\mathbf{P})_{*}(B)=1-(\mathbf{P})^{*}\left(\Lambda_{0 \Sigma} \backslash B\right)$.

For a sequence of random variables $Z_{n}: \Upsilon \rightarrow R^{1}\left(Z_{n}\right.$ are not necessary measurable ) we say that $\liminf _{n \rightarrow \infty} Z_{n} \geqslant c$ inner almost surely $\left(a . s_{*}\right)$, if there are measurable random variables $\Delta_{n}$, such that $\Delta_{n} \leqslant Z_{n}$ and $\mathbf{P}\left(\liminf _{n \rightarrow \infty} \Delta_{n} \geqslant c\right)=1$.

We say that $\limsup _{n \rightarrow \infty} Z_{n} \leqslant c$ inner almost surely $\left(a . s^{*}\right.$.) if $\liminf _{n \rightarrow \infty}-Z_{n} \geqslant-$ ca.s. $s_{*}$.

We say that $\limsup _{n \rightarrow \infty} Z_{n}=-\infty$ outer almost surely $\left(a . s^{*}\right.$.) if $\liminf _{n \rightarrow \infty}-Z_{n} \geqslant$ $-c a . s_{*}$ for all $c>0$. 
2.4. Conditional moderate deviation principle for bootstrap empirical measures. Theorem 2.1 given below shows that MDP holds almost surely (a.s.) for the conditional distribution of bootstrap empirical measure given empirical probability measure. In this setup we allow the sample size $k=k_{n}$ of the bootstrap to have values different from $n$.

The results will be provided in terms of the $\tau_{\Theta}$-topologies.

For each $t>2$ define the set $\Theta=\Theta_{t}$ of real functions $f: S \rightarrow R^{1}$ such that $E\left[|f(X)|^{t}\right]<\infty$.

For decreasing function $h: R_{+}^{1} \rightarrow R_{+}^{1}$ and $t \geqslant 2$ define the set $\Theta=\Theta_{t, h}$ of real functions $f$ such that

$$
P\left(|f(X)|>s^{-1}\right)<h(s), \quad s>0
$$

and

$$
E\left[|f(X)|^{t}\right]<\infty \text {. }
$$

Let $X_{1}^{*}, \ldots, X_{k_{n}}^{*}$ be i.i.d.r.v.'s having pm $\hat{P}_{n}$. Denote $P_{k_{n}}^{*}$ the empirical probability measure of $X_{1}^{*}, \ldots, X_{k_{n}}^{*}$. Suppose that $\frac{k_{n}}{n}<c<\infty$ and $k_{n} \rightarrow \infty$ as $n \rightarrow \infty$.

2.1. Let a decreasing sequence $a_{n}>0, a_{n} \rightarrow 0, a_{n+1} / a_{n} \rightarrow 1, k_{n} a_{n}^{2} \rightarrow \infty$ as $n \rightarrow \infty$ be provided. Let

$$
\sum_{n=1}^{\infty} h\left(c a_{n}\right)<\infty
$$

for all $c>0$.

Let $\Omega_{0} \subset \Lambda_{0 \Theta_{2, h}}$. Then there hold

$$
\liminf _{n \rightarrow \infty}\left(k_{n} a_{n}^{2}\right)^{-1} \ln \left(\widehat{\mathbf{P}}_{n}\right)_{*}\left(\mathbf{P}_{k_{n}}^{*} \in \widehat{\mathbf{P}}_{n}+a_{n} \Omega_{0}\right) \geqslant-\rho_{0}^{2}\left(\mathfrak{i n t}\left(\Omega_{0}\right), \mathbf{P}\right) \quad \text { a. } s_{*}
$$

and

$$
\limsup _{n \rightarrow \infty}\left(k_{n} a_{n}^{2}\right)^{-1} \ln \left(\widehat{\mathbf{P}}_{n}\right)^{*}\left(\mathbf{P}_{k_{n}}^{*} \in \widehat{\mathbf{P}}_{n}+a_{n} \Omega_{0}\right) \leqslant-\rho_{0}^{2}\left(\mathfrak{c l}\left(\Omega_{0}\right), \mathbf{P}\right) \quad a . s^{*},
$$

where the closure and the interior of the set $\Omega_{0}$ in (2.4) and (2.5) are considered with respect to $\tau_{\Theta_{2, h}}$-topology. The outer probability measure $\left(\widehat{\mathbf{P}}_{n}\right)^{*}$ and the inner probability measure $\left(\widehat{\mathbf{P}}_{n}\right)_{*}$ are considered with respect to $\sigma_{\Theta_{2, h}}$-algebra.

Let $\Omega_{0} \subset \Lambda_{0 \Theta_{t}}, t>2$ and let $a_{n}=o\left(n^{-1 / t}\right)$. Then (2.4) and (2.5) are valid if $\operatorname{int}\left(\Omega_{0}\right)$ and $\mathfrak{c l}\left(\Omega_{0}\right)$ are considered with respect to $\tau_{\Theta_{t}}$-topology. Outer probability measure $\left(\widehat{\mathbf{P}}_{n}\right)^{*}$ and inner probability measure $\left(\widehat{\mathbf{P}}_{n}\right)_{*}$ are considered with respect to $\sigma_{\Theta_{t}}$-algebra.

\subsection{Rates of convergence in conditional moderate deviation principle.}

2.2. Let a decreasing sequence $a_{n}>0, a_{n} \rightarrow 0, a_{n+1} / a_{n} \rightarrow 1, k_{n} a_{n}^{2} \rightarrow \infty$ as $n \rightarrow \infty$ be given. Let function $h: R_{+}^{1} \rightarrow R_{+}^{1}$ be such that

$$
\lim _{n \rightarrow \infty} n h\left(c a_{n}\right)=0
$$

for each $c>0$. Let $\Omega_{0} \subset \Lambda_{0 \Theta_{t, h}}, t>2$. Then for any

$$
\epsilon>0 \text { and } n>n_{0}\left(\epsilon,\left\{k_{i}\right\}_{i=1}^{\infty}, \Omega_{0}\right)
$$

there hold

$$
\left(k_{n} a_{n}^{2}\right)^{-1} \log \left(\widehat{\mathbf{P}}_{n}\right)_{*}\left(\mathbf{P}_{k_{n}}^{*} \in \widehat{\mathbf{P}}_{n}+a_{n} \Omega_{0}\right) \geqslant-\rho_{0}^{2}\left(\mathfrak{i n t}\left(\Omega_{0}\right), \mathbf{P}\right)-\epsilon
$$

and, if $\rho_{0}^{2}\left(\mathfrak{c l}_{\Theta_{t, h}}\left(\Omega_{0}\right), \mathbf{P}\right)<\infty$ additionally, then

$$
\left(k_{n} a_{n}^{2}\right)^{-1} \log \left(\widehat{\mathbf{P}}_{n}\right)^{*}\left(\mathbf{P}_{k_{n}}^{*} \in \widehat{\mathbf{P}}_{n}+a_{n} \Omega_{0}\right) \leqslant-\rho_{0}^{2}\left(\mathfrak{c l}\left(\Omega_{0}\right), \mathbf{P}\right)+\epsilon
$$

on the sets of events having the inner probabilities more than $\kappa_{n}=\kappa_{n}\left(\epsilon, \Omega_{0}\right)=$ $1-C\left(\epsilon, \Omega_{0}\right)\left[\beta_{1 n}+\beta_{2 n}\right]$ where $\beta_{1 n}=n h\left(\frac{a_{n}}{\epsilon C_{1}\left(\epsilon, \Omega_{0}\right)}\right)$ and $\beta_{2 n}=C_{2}\left(\epsilon, \Omega_{0}\right) n^{1-t / 2}$. 
If $\rho_{0}^{2}\left(\mathfrak{c l}\left(\Omega_{0}\right), \mathbf{P}\right)=\infty$, then for any $L>0$

$$
\left(k_{n} a_{n}^{2}\right)^{-1} \log \left(\widehat{\mathbf{P}}_{n}\right)^{*}\left(\mathbf{P}_{k_{n}}^{*} \in \widehat{\mathbf{P}}_{n}+a_{n} \Omega_{0}\right) \leqslant-L
$$

on the sets of events having the inner probabilities more than $\kappa_{1 n}=\kappa_{1 n}\left(L, \Omega_{0}\right)=$ $1-C\left(L, \Omega_{0}\right)\left[\beta_{1 n}+\beta_{2 n}\right]$ with $\beta_{1 n}=n h\left(\frac{a_{n}}{C_{1}\left(L, \Omega_{0}\right)}\right)$ and $\beta_{2 n}=C_{2}\left(L, \Omega_{0}\right) n^{1-t / 2}$.

3. Moderate DeViation PRINCIPLE For the COMMON Distributions of EMPIRICAL MEASURES AND BOOTSTRAP EMPIRICAL MEASURES

In section we prove MDP for the common distribution of $\left(\mathbf{P}_{k_{n}}^{*}-\widehat{\mathbf{P}}_{n}\right) \times\left(\widehat{\mathbf{P}}_{n}-\mathbf{P}\right)$. We suppose that $k_{n} / n \rightarrow \nu$ as $n \rightarrow \infty$.

3.1. Basic definitions. Define sequence $b_{n}$ such that

$$
\left.\begin{array}{ll}
b_{n} & \rightarrow 0 \\
n b_{n}^{2} & \rightarrow \infty \\
\frac{b_{n}}{b_{n+1}} & \rightarrow 1
\end{array}\right\} \quad \text { as } \quad n \rightarrow \infty .
$$

MDP is provided in terms of the $\tau_{\Phi}$-topology with the set $\Phi$ of measurable functions $f$ such that

$$
\lim _{n \rightarrow \infty} \frac{1}{n b_{n}^{2}} \log \left(n \mathbf{P}\left(|f(X)|>b_{n}^{-1}\right)\right)=-\infty .
$$

Define the $\tau_{\Phi}$-topology in $\Lambda_{\Phi}^{2}$ and $\Lambda_{0 \Phi}^{2}$ as the product of $\tau_{\Phi}$-topologies.

For any $\overline{\mathbf{G}}=\mathbf{G}_{2} \times \mathbf{G}_{1} \in \Lambda_{0}^{2}$ the rate function equals

$$
\rho_{0 b}^{2}(\overline{\mathbf{G}}, \mathbf{P})=\nu \rho_{0}^{2}\left(\mathbf{G}_{2}, \mathbf{P}\right)+\rho_{0}^{2}\left(\mathbf{G}_{1}, \mathbf{P}\right) .
$$

For any set $\bar{\Omega}_{0} \subset \Lambda_{0 \Phi}^{2}$ denote

$$
\rho_{0 b}^{2}\left(\bar{\Omega}_{0}, \mathbf{P}\right)=\inf \left\{\rho_{0 b}^{2}(\overline{\mathbf{G}}, \mathbf{P}): \overline{\mathbf{G}} \in \bar{\Omega}_{0}\right\} .
$$

We fix signed measures $\mathbf{H}, \mathbf{H}_{n} \in \Lambda_{0 \Phi}$ satisfying the following assumptions.

A. There hold

$$
\mathbf{P}_{n}=\mathbf{P}+b_{n} \mathbf{H}_{n} \in \Lambda_{\Phi}, \quad \mathbf{P}+b_{n} \mathbf{H} \in \Lambda_{\Phi}
$$

and $\mathbf{H}_{n} \rightarrow \mathbf{H}$ as $n \rightarrow \infty$ in the $\tau_{\Phi}$-toplogy.

B1. For any $f \in \Phi$

$$
\limsup _{n \rightarrow \infty} \sup _{m}\left(n b_{n}^{2}\right)^{-1} \log \left(n b_{n} \int \chi\left(|f(x)|>b_{n}^{-1}\right) d\left|\mathbf{H}_{m}\right|\right)=-\infty .
$$

Define the signed measure $\mathbf{O} \in \Lambda_{0 \Phi}$, such that $\mathbf{O}(A)=0$ for any set $A \in \mathcal{F}$. For all $\mathbf{G} \in \Lambda_{0 \Phi}$ denote $\widetilde{\mathbf{G}}=\mathbf{O} \times \mathbf{G}$.

3.1. Assume $\mathrm{A}$ and $\mathrm{B} 1$. Let $\bar{\Omega}_{0} \subset \Lambda_{0 \Phi}^{2}$ be $\sigma_{\Phi}$-measurable set in $\Lambda_{0 \Phi}^{2}$. Then the following MDP holds

$$
\begin{aligned}
\liminf _{n \rightarrow \infty}\left(n b_{n}^{2}\right)^{-1} \log \mathbf{P}_{n}\left(\left(\mathbf{P}_{n}^{*}-\widehat{\mathbf{P}}_{n}\right) \times\left(\widehat{\mathbf{P}}_{n}-\mathbf{P}_{0}\right) \in b_{n} \bar{\Omega}_{0}\right) & \\
& \geqslant-\rho_{0 b}^{2}\left(\mathfrak{i n t}\left(\bar{\Omega}_{0}-\widetilde{\mathbf{H}}\right), \mathbf{P}\right)
\end{aligned}
$$

and

$$
\begin{aligned}
\limsup _{n \rightarrow \infty}\left(n b_{n}^{2}\right)^{-1} \log \mathbf{P}_{n}\left(\left(\mathbf{P}_{n}^{*}-\widehat{\mathbf{P}}_{n}\right) \times\left(\widehat{\mathbf{P}}_{n}-\mathbf{P}\right) \in b_{n} \bar{\Omega}_{0}\right) & \\
& \leqslant-\rho_{0 b}^{2}\left(\mathfrak{c l}\left(\bar{\Omega}_{0}-\widetilde{\mathbf{H}}\right), \mathbf{P}\right) .
\end{aligned}
$$


Bolthausen [5] has proved the Donsker-Varadhan LDP [1] when the laws of random variables converge weakly and a uniform exponential integration condition is satisfied. Theorem 3.1 can be considered as a version of these results.

Remark 2.1. In hypothesis testing, the type II error probabilities are often analyzed for the alternatives $\mathbf{P}_{n}$ converging to the hypothesis $\mathbf{P}$. Theorem 3.1 allows to study moderate deviation probabilities for this setup. The analysis of importance sampling efficiency is also based on MDP with a sequence of p.m.'s $\mathbf{P}_{n}$ converging to p.m. $\mathbf{P}$ (see [15]). Naturally, if we suppose that $\mathbf{H}_{n}, \mathbf{H}$ are absent, we get usual form of MDP.

The modern form of LDP-MDP (see. [8, 16, 21]) covers the case of unmeasurable sets $\bar{\Omega}_{0}$ and is provided in terms of outer and inner probabilities (see Theorems 2.2 and 2.1). Theorem 3.1 can be also provided in such a form.

Theorem 3.2 provided below shows that we can not make significantltly larger the zones of moderate deviation probabilities in Theorem 3.1

3.2. Let random variable $Y=|f(X)|$ satisfies (3.1). Let sequences $r_{n}$ and $e_{n}$ be such that $b_{n}^{-1}<r_{n}, b_{n}^{-1} e_{n} \rightarrow \infty, n e_{n} / r_{n} \rightarrow \infty$ as $n \rightarrow \infty$ and

$$
\begin{gathered}
\lim _{n \rightarrow \infty}\left(n e_{n}^{2}\right)^{-1} \log \left(n \mathbf{P}\left(Y>r_{n}\right)\right)=0, \\
\lim _{n \rightarrow \infty}\left(r_{n} e_{n}\right)^{-1} \log \frac{n e_{n}}{r_{n}}=0 .
\end{gathered}
$$

Let $Y_{1}, \ldots, Y_{n}$ be independent copies of $Y$ and let $Y_{1}^{*}, \ldots, Y_{n}^{*}$ be bootstrap sample obtained from $Y_{1}, \ldots, Y_{n}$. Then

$$
\lim _{n \rightarrow \infty}\left(n e_{n}^{2}\right)^{-1} \log \mathbf{P}\left(\sum_{i=1}^{n} Y_{i}^{*}>n e_{n}\right)=0 .
$$

Proof of Theorem 3.2 are provided in section 7 .

Example. Let $\mathbf{P}(Y>t)=\exp \left\{-t^{\gamma}\right\}, 0<\gamma<1$. Then $b_{n}=o\left(n^{-\frac{1}{2+\gamma}}\right)$. By straightforward calculations, we get that (3.2), (3.3) hold for any sequence $r_{n}=$ $n^{\frac{1}{2+\gamma}} f_{n}, e_{n}=n^{-\frac{1}{2+\gamma}} f_{n}^{\frac{\gamma}{2}-\delta}$, with $(\log n)^{\frac{1}{1+\frac{\gamma}{2}-\delta}}<<f_{n}<<n^{\frac{\gamma}{(2+\gamma)(1+\delta)}}$ and $0<\delta<$ $\frac{\gamma}{2}$. Therefore we can not improve significantly the moderate deviation zone in Theorem 3.1 for this asymptotic of $\mathbf{P}(Y>t)$.

3.2. Moderate deviation principle for empirical measure. Theorem 3.3 provided below can be considered as a version of moderate deviation principle established in [2] for empirical processes. In such a form this MDP has been proved in [15] and is provided here for comparison with the bootstrap results.

Define the set $\Psi$ of measurable functions $f: S \rightarrow R^{1}$ such that

$$
\lim _{n \rightarrow \infty}\left(n d_{n}^{2}\right)^{-1} \log \left(n \mathbf{P}\left(|f(X)|>n d_{n}\right)\right)=-\infty
$$

where $d_{n} \rightarrow 0, n d_{n}^{2} \rightarrow \infty, d_{n+1} / d_{n} \rightarrow 1$ as $n \rightarrow \infty$.

Suppose the following.

B2. For each $f \in \Psi$, there holds

$$
\lim _{n \rightarrow \infty}\left(n d_{n}^{2}\right)^{-1} \sup _{m} \log \left(n d_{n} \int \chi\left(|f(x)|>n d_{n}\right) d\left|H_{m}\right|\right)=-\infty .
$$

Using the reasoning of Lemma 2.5 in [14, we get that B1 and B2 imply

$$
\sup _{m} \int f^{2} d\left|H_{m}\right|<\infty
$$

and (3.1) or (3.4) implies

$$
\int f^{2} d \mathbf{P}<\infty
$$


In Lemma 2.5 in [14, (3.6) has been proved, if $d_{n}$ is decreasing and $n^{1 / 2} d_{n}$ is increasing. Since $d_{n} / d_{n-1} \rightarrow 1$ as $n \rightarrow \infty$ we can choose a subsequence $d_{n_{k}}$ such that $n_{k}^{1 / 2} d_{n_{k}}$ is increasing and $d_{n_{k}} / d_{n_{k-1}} \rightarrow 1$ as $k \rightarrow \infty$. After that we can choose a subsequence $d_{n_{k_{i}}}$ such that $d_{n_{k_{i}}}$ is decreasing and $d_{n_{k_{i}}} / d_{n_{k_{i-1}}} \rightarrow 1$ as $i \rightarrow \infty$. Implementing to the subsequence $d_{n_{k_{i}}}$ the same reasoning as in the proof of Lemma 2.5 in [14] we get (3.6) without assuming that the sequences $d_{n}$ and $n^{1 / 2} d_{n}$ are monotone.

3.3. Assume $\mathrm{A}$ with $\Phi=\Psi$ and $\mathrm{B} 2$. Let the set $\Omega_{0}$ is the $\sigma_{\Psi}$-measurable subset of $\Lambda_{0 \Psi}$. Then MDP holds

$$
\liminf _{n \rightarrow \infty}\left(n d_{n}^{2}\right)^{-1} \log \mathbf{P}_{n}\left(\widehat{\mathbf{P}}_{n} \in \mathbf{P}+d_{n} \Omega_{0}\right) \geqslant-\rho_{0}^{2}\left(\mathfrak{i n t}\left(\Omega_{0}-\mathbf{H}\right), \mathbf{P}_{0}\right)
$$

and

$$
\limsup _{n \rightarrow \infty}\left(n d_{n}^{2}\right)^{-1} \log \mathbf{P}_{n}\left(\widehat{\mathbf{P}}_{n} \in \mathbf{P}+d_{n} \Omega_{0}\right) \leqslant-\rho_{0}^{2}\left(\mathfrak{c l}\left(\Omega_{0}-\mathbf{H}\right), \mathbf{P}_{0}\right)
$$

Example. Let $\mathbf{E}\left[\exp \left\{c\left|f\left(X_{1}\right)\right|^{\gamma}\right\}\right]<\infty$, for all $f \in \Theta$ with $\gamma>0$. Then there hold

$$
b_{n}=o\left(n^{-\frac{1}{1+\gamma}}\right), \quad d_{n}=o\left(n^{-\frac{1-\gamma}{2-\gamma}}\right) \quad \text { and } \quad a_{n}=o\left(|\log n|^{-\gamma}\right) .
$$

Therefore conditional MDP holds for significantly wider zone than MDP for empirical measures.

\section{Moderate Deviation probabilities of Statistical functionals}

For statistical functionals the technique of Freshet and Hadamard derivatives (see [15] and [16]) works for the proofs of MDPs to the same extent as in the proofs of asymptotic normality.

4.1. Differentiable statistical functionals. For statistical functionals having the Freshet derivatives MDP has been studied in [15]. For functionals having the Hadamard derivatives MDP technique has been developed in 16. Instead of convergence in the weak topology, in these results the differentiability of statistical functionals in some metric space is supposed. If $S=R^{d}$, the Kolmogorov-Smirnov metric on the set of distribution functions is continuous in the $\tau$-topology (see [17]). Thus the functionals continuous in Kolmogorov-Smirnov metric satisfies MDP. This approach has been implemented in [15] for the proof of MDP for $L$ and $M$ statistics having the Freshet derivatives. In [16], the Hadamard differentiability in KS-metric allows to derive MDP for Kaplan-Meier estimator, empirical quantile processes and empirical copula functions. The continuiuty of KS-metric in the $\tau$-topology allows to replace MDP for empirical processes with MDP for empirical probability measures in the reasoning. MDP for the bootstrap empirical quantile processes and MDP for the bootstrap empirical copula functions provided in the subsequent subsections follows straightforwardly from the continuiuty of KS-distance in the $\tau$-topology, Theorem 2.1 and Theorem 3.1 in [16].

In [15, we prove that Kolmogorov-Smirnov metric having some weight function is continuous in the $\tau_{\Psi}$-topology. A version of this result for the $\tau_{\Theta_{t}}$-topology will be provided in the subsection.

Suppose that

$$
\int|x|^{t \kappa} d \mathbf{P}<\infty
$$

with $t>2, \kappa>0$.

Define the set $\Upsilon$ of measurable functions $f: R^{d} \rightarrow R^{1}$ such that

$$
|f(x)| \leqslant C\left(1+|x|^{\kappa}\right), \quad x \in R^{d} .
$$


Define the set $\Lambda_{\kappa}$ of all probability measures $\mathbf{Q}$ such that

$$
\int|x|^{\kappa} d \mathbf{Q}<\infty
$$

Let $F(x), x \in R^{d}$, be distribution function of probability measure $\mathbf{P}$.

For any $\mathbf{P}$ and $\mathbf{Q}$ define the distance

$$
\rho_{\kappa}(\mathbf{P}, \mathbf{Q})=\sup _{x \in R^{d}}\left|F_{Q}(x)-F_{P}(x)\right|\left(1+|x|^{\kappa}\right)
$$

where $F_{Q}$ and $F_{P}$ stand for c.d.f.'s of probability measures $\mathbf{Q}$ and $\mathbf{P}$ respectively.

Define the $\rho_{\kappa}$-topology in $\Lambda_{\kappa}$ generated by the distance $\rho_{\kappa}$.

4.1. The $\rho_{\kappa}$-topology is coaser than the $\tau_{\Upsilon}$-topology.

The proof of Theorem 4.1 is akin to the proof of Lemma 4.1 in [15] and is omitted.

4.2. Bootstrap empirical quantile processes. Denote $D[a, b],-\infty<a<b<$ $\infty$ the Banach space of all right continuous with left-hand limits functions $f$ : $[a, b] \quad \rightarrow \quad R^{1}$ equipped with uniform norm. Let $F(x), x \in(a, b)$ - be distribution function of independent identically distributed random variables $X_{1}, \ldots, X_{n}$. Denote $\widehat{F}_{n}$ and $F_{k_{n}}^{*}$ respectively empirical distribution functions of $X_{1}, \ldots, X_{n}$ and $X_{1}^{*}, \ldots, X_{k_{n}}^{*}$.

For any distribution function $G(x), x \in(a, b)$, and any $p \in(0,1)$, denote $(G)^{-1}(p)=\inf \{x: G(x) \geqslant p\}$.

4.2. Let $a_{n}>0$ be decreasing sequence such that $a_{n} \rightarrow 0, a_{n+1} / a_{n} \rightarrow 1, k_{n} a_{n}^{2} \rightarrow \infty$ as $n \rightarrow \infty$. Let fixed values of $p$ and $q$ with $0<p<q<1$ be provided. Let $F$ have continuous and positive density on interval $\left[(F)^{-1}(p)-\epsilon,(F)^{-1}(q)+\epsilon\right]$ with $\epsilon>0$. Then, for any set $\Omega \subset D\left((F)^{-1}(p),(F)^{-1}(q)\right)$, we have

$$
\liminf _{n \rightarrow \infty}\left(k_{n} a_{n}^{2}\right)^{-1} \log \left(\widehat{\mathbf{P}}_{n}\right)_{*}\left(\left(F_{k_{n}}^{*}\right)^{-1}-\left(\widehat{F}_{n}\right)^{-1} \in a_{n} \Omega\right) \geqslant-I_{q}(\mathfrak{i n t}(\Omega)) \quad a . s_{*}
$$

and

$$
\limsup _{n \rightarrow \infty}\left(k_{n} a_{n}^{2}\right)^{-1} \log \left(\widehat{\mathbf{P}}_{n}\right)^{*}\left(\left(F_{k_{n}}^{*}\right)^{-1}-\left(\widehat{F}_{n}\right)^{-1} \in a_{n} \Omega\right) \leqslant-I_{q}(\mathfrak{c l}(\Omega)) \quad a . s^{*},
$$

where for any set $\Psi \subset D\left((F)^{-1}(p)-\epsilon,(F)^{-1}(q)+\epsilon\right)$

$$
\begin{aligned}
& I_{q}(\Psi)=\inf \left\{\rho_{0}^{2}(\mathbf{Q}, \mathbf{P}): \mathbf{Q} \in \Lambda_{0 \Theta_{2}}, q=\frac{d \mathbf{Q}}{d \mathbf{P}},-\frac{q\left((F)^{-1}(x)\right)}{f\left((F)^{-1}(x)\right)}=\phi(x),\right. \\
& \phi(x) \in \Psi, x \in[p, q]\} .
\end{aligned}
$$

4.3. Let $b_{n}>0$ be decreasing sequence such that $b_{n} \rightarrow 0, b_{n+1} / b_{n} \rightarrow 1, k_{n} b_{n}^{2} \rightarrow \infty$ as $n \rightarrow \infty$. Let $k_{n} / n \rightarrow \nu$ as $n \rightarrow \infty$. Let $F$ satisfy the conditions of Theorem 4.2 . Then, for any sets

$$
\Omega_{1} \subset D\left((F)^{-1}(p),(F)^{-1}(q)\right) \text { and } \Omega_{2} \subset D\left((F)^{-1}(p),(F)^{-1}(q)\right),
$$

we have

$$
\begin{aligned}
& \liminf _{n \rightarrow \infty}\left(n b_{n}^{2}\right)^{-1} \ln (\mathbf{P})_{*}\left(\left(F_{k_{n}}^{*}\right)^{-1}-\left(\widehat{F}_{n}\right)^{-1} \in b_{n} \Omega_{2},\left(\widehat{F}_{n}\right)^{-1}-(F)^{-1} \in b_{n} \Omega_{1}\right) \\
& \geqslant-\nu I_{q}\left(\mathfrak{i n t}\left(\Omega_{2}\right)\right)+I_{q}\left(\mathfrak{i n t}\left(\Omega_{1}\right)\right)
\end{aligned}
$$

and

$$
\begin{aligned}
& \limsup _{n \rightarrow \infty}\left(n b_{n}^{2}\right)^{-1} \ln (\mathbf{P})^{*}\left(\left(F_{k_{n}}^{*}\right)^{-1}-\left(\widehat{F}_{n}\right)^{-1} \in b_{n} \Omega_{2},\left(\widehat{F}_{n}\right)^{-1}-(F)^{-1} \in b_{n} \Omega_{1}\right) \\
& \leqslant-\nu I_{q}\left(\mathfrak{c l}\left(\Omega_{2}\right)\right)+I_{q}\left(\mathfrak{c l}\left(\Omega_{1}\right)\right) .
\end{aligned}
$$


4.3. Bootstrap empirical copula processes. Let $\left(X_{1}, Y_{1}\right)$, $\ldots,\left(X_{n}, Y_{n}\right)$ be independent identically distributed random vectors having probability measure $\mathbf{P}$ defined on $(a, b) \times(c, d) \supset R^{2}$. Let $H$ be distribution function of P. The empirical estimator of copula function $C(u, v)=H\left((F)^{-1}(u),(G)^{-1}(v)\right)$ is defined as $\widehat{C}_{n}(u, v)=\widehat{H}_{n}\left(\left(\widehat{F}_{n}\right)^{-1}(u),\left(\widehat{G}_{n}\right)^{-1}(v)\right)$ where $\widehat{H}_{n}$ and $\widehat{F}_{n}, \widehat{G}_{n}$ are respectively the joint and marginal distribution functions of observations. The bootstrap empirical copula function is defined similarly $C_{n}^{*}(u, v)=H_{n}^{*}\left(\left(F_{n}^{*}\right)^{-1}(u)\right.$, $\left.\left(G_{n}^{*}\right)^{-1}(v)\right)$ using the observations $\left(X_{1}^{*}, Y_{1}^{*}\right), \ldots,\left(X_{k_{n}}^{*}, Y_{k_{n}}^{*}\right)$. Here $\left(X_{1}^{*}, Y_{1}^{*}\right), \ldots,\left(X_{k_{n}}^{*}, Y_{k_{n}}^{*}\right)$ are distributed with respect to empirical probability measure $\widehat{\mathbf{P}}_{n}$ generated by the observations $\left(X_{1}, Y_{1}\right), \ldots,\left(X_{n}, Y_{n}\right)$.

For any set $S$ denote $l_{\infty}(S)$ linear space of all maps $z: S \rightarrow R^{1}$ having the norm $\|z\|=\sup _{s \in S}|z(s)|$.

4.4. Let $a_{n}>0$ be decreasing sequence such that $a_{n} \rightarrow 0, a_{n+1} / a_{n} \rightarrow 1, k_{n} a_{n}^{2} \rightarrow \infty$ as $n \rightarrow \infty$. Let $0<p_{1}<q_{1}<1$ and $0<p_{2}<q_{2}<1$ be fixed. Suppose that $F$ and $G$ are continuously differentiable on the intervals $\left[(F)^{-1}\left(p_{1}\right)-\epsilon,(F)^{-1}\left(q_{1}\right)+\epsilon\right]$ and $\left[(G)^{-1}\left(p_{2}\right)-\epsilon,(G)^{-1}\left(q_{2}\right)+\epsilon\right]$ respectively and have strictly positive densities $f$ and $g$ respectively with $\epsilon>0$. Suppose that there are continuous derivatives $\partial H / \partial x$ and $\partial H / \partial y$ on the product of intervals

$$
\left[(F)^{-1}\left(p_{1}\right)-\epsilon,(F)^{-1}\left(q_{1}\right)+\epsilon\right] \times\left[(G)^{-1}\left(p_{2}\right)-\epsilon,(G)^{-1}\left(q_{2}\right)+\epsilon\right] .
$$

Then, for any set $\Omega \subset l_{\infty}\left(\left[p_{1}, q_{1}\right] \times\left[p_{2}, q_{2}\right]\right)$, we have

$$
\liminf _{n \rightarrow \infty}\left(k_{n} a_{n}^{2}\right)^{-1} \log \left(\widehat{\mathbf{P}}_{n}\right)_{*}\left(C_{k_{n}}^{*}-\widehat{C}_{n} \in a_{n} \Omega\right) \geqslant-I_{C}(\mathfrak{i n t}(\Omega)) \quad a . s_{*}
$$

and

$$
\limsup _{n \rightarrow \infty}\left(k_{n} a_{n}^{2}\right)^{-1} \log \left(\widehat{\mathbf{P}}_{n}\right)^{*}\left(C_{k_{n}}^{*}-\widehat{C}_{n} \in a_{n} \Omega\right) \leqslant-I_{C}(\mathfrak{c l}(\Omega)) \quad a . s^{*},
$$

where, for any set $\Psi \subset l_{\infty}\left(\left[p_{1}, q_{1}\right] \times\left[p_{2}, q_{2}\right]\right)$, there holds

$$
\begin{array}{r}
I_{C}(\Psi)=\inf \left\{\rho_{0}(\mathbf{Q}): q=\frac{d \mathbf{Q}}{d \mathbf{P}}, \mathbf{Q} \in \Lambda_{0 \Theta_{2}}, \Phi_{H}^{\prime}(\alpha)=\phi, \phi \in \Psi,\right. \\
\left.\alpha(s, t)=\int_{-\infty}^{s} \int_{-\infty}^{t} q(x, y) H(d x, d y)\right\},
\end{array}
$$

with $\Phi_{H}^{\prime}$ defined by

$$
\begin{gathered}
\Phi_{H}^{\prime}(\alpha)(u, v)=\alpha\left((F)^{-1}(u),(G)^{-1}(v)\right) \\
-\frac{\partial H}{\partial x}\left((F)^{-1}(u),(G)^{-1}(v)\right) \frac{\alpha\left((F)^{-1}(u), \infty\right)}{f\left((F)^{-1}(u)\right)} \\
-\frac{\partial H}{\partial y}\left((F)^{-1}(u),(G)^{-1}(v)\right) \frac{\alpha\left(\infty,(G)^{-1}(v)\right)}{g\left((G)^{-1}(v)\right)} .
\end{gathered}
$$

Remark. Versions of Theorem 4.2 and 4.4 can be provided also in terms of convergence on probability (see Theorem 2.2).

\section{Proofs of Theorems 2.1 and 2.2}

We begin with the proof of Theorem 2.2. The reasoning are based on the proof LDP for empirical measures proposed in 8. For any $r>0$ define the set $\Gamma_{0 r}=$ $\left\{G: \rho_{0}^{2}(G: P)<r, G \in \Lambda_{0 \Theta}\right\}$. 
5.1. There hold

(i) $\Gamma_{0 r} \subset \Lambda_{0 \Theta}$,

(ii) $\Gamma_{0 r}$ is $\tau_{\Theta}$-compact and sequentially $\tau_{\Theta}$-compact set in $\Lambda_{0 \Theta}$,

(iii) the $\tau$ and $\tau_{\Theta}$-topologies coincide in $\Gamma_{0 r}$.

Proof. The reasoning are akin to the proof of Lemma 2.1 in [13]. For any signed measure $\mathbf{G} \in \Gamma_{0 r}$, any measurable set $A \subseteq S$ and each $\phi \in \Theta$, we have

$$
\int_{A}|\phi| d|\mathbf{G}| \leqslant \alpha\left(\int_{A} \phi^{2} d \mathbf{P}\right)+\alpha^{-1}\left(\int_{A}\left(\frac{d \mathbf{G}}{d \mathbf{P}}\right)^{2} d \mathbf{P}\right)^{2} d \mathbf{P}
$$

for all $\alpha>0$. By the definition of $\Gamma_{0 r}$, this implies (i), if $A=S$.

Fix $\epsilon>0$. Let $\alpha=r / \epsilon$ and let $n=n(\epsilon)$ be such that

$$
\frac{r}{\epsilon}\left(\int_{|\phi|>n} \phi^{2} d \mathbf{P}\right)<\epsilon \text {. }
$$

Then

$$
\alpha^{-1} \int_{|\phi|>n}\left(\frac{d \mathbf{G}}{d \mathbf{P}}\right)^{2} d \mathbf{P} \leqslant \epsilon .
$$

Therefore, by (5.1), we get

$$
\int|\phi| d|\mathbf{G}|-\int_{|\phi|<n}|\phi| d|\mathbf{G}|<2 \epsilon
$$

Hence the map $\Gamma_{0 r} \ni \mathbf{G} \rightarrow \int \phi d \mathbf{G}$ is $\tau$-continuous as continuous limit of functions

$$
\int_{\left|\phi_{1}\right|<n} \phi d \mathbf{G} \text {. }
$$

Therefore the $\tau$ and $\tau_{\Theta}$-topologies coincide in $\Gamma_{0 r}$.

Since the sets $\Gamma_{0 r}$ are $\tau$-compact and sequentially $\tau$-compact (see [2, 4]), these sets are $\tau_{\Theta}$-compact and sequentially $\tau_{\Theta}$-compact. This completes the proof of Lemma 5.1

We begin with the proof of upper bound (2.8). Denote $\eta=\rho_{0}^{2}\left(\mathfrak{c l}\left(\Omega_{0}\right), \mathbf{P}\right)$ and fix $\delta, 0<2 \delta<\eta$. It is clear that $\Gamma_{0, \eta-\delta} \subset \Lambda_{0 \Theta} \backslash \Omega_{0}$.

For any $f_{1}, \ldots, f_{l} \in \Theta, \mathbf{G} \in \Lambda_{0 \Theta}$ and $\gamma>0$, denote

$$
U\left(f_{1}, \ldots, f_{l}, \mathbf{G}, \gamma\right)=\left\{\mathbf{R}:\left|\int f_{i} d(\mathbf{R}-\mathbf{G})\right|<\gamma, \mathbf{R} \in \Lambda_{0 \Theta}, 1 \leqslant i \leqslant l\right\} .
$$

Define the linear space

$$
\widetilde{\Lambda}_{0 \Theta}=\left\{\mathbf{G}: \mathbf{G}=\sum_{i=1}^{k} \lambda_{i} \mathbf{G}_{i}, \mathbf{G}_{i} \in \Lambda_{0 \Theta}, \lambda_{i} \in R^{1}, 1 \leqslant i \leqslant k, k=1,2, \ldots\right\} .
$$

Define the $\tau_{\Theta}$-topology in $\widetilde{\Lambda}_{0 \Theta}$. It is clear that $\Lambda_{0 \Theta} \subset \widetilde{\Lambda}_{0 \Theta}$.

Since $\Lambda_{0 \Theta}$ is Hausdorff topological space, then the space $\Lambda_{0 \Theta}$ is regular (see Theorem B2 in [10]). Therefore, for each $\mathbf{G} \in \Gamma_{0, \eta-\delta}$, there is open set $U\left(f_{1}, \ldots, f_{l}, \mathbf{G}, \gamma\right) \subset$ $\Lambda_{0 \Theta} \backslash \mathrm{cl}\left(\Omega_{0}\right)$. The set $\Gamma_{0, \eta-\delta}$ is compact. Therefore there is finite covering of $\Gamma_{0, \eta-\delta}$ by the sets

$$
U_{1}=U\left(f_{11}, \ldots, f_{1 l_{1}}, \mathbf{G}_{1}, c_{1}\right), \ldots, U_{m}=U\left(f_{m 1}, \ldots, f_{m l_{m}}, \mathbf{G}_{m}, c_{m}\right),
$$

where $f_{i j} \in \Theta, \mathbf{G}_{i} \in \Lambda_{0 \Theta} 1 \leqslant j \leqslant l_{i}, 1 \leqslant i \leqslant m$. Denote $U=\cup_{i=1}^{m} U_{i}$.

Therefore, for the proof of (2.8), it suffices to estimate left-hand side

$$
\widehat{\mathbf{P}}_{n}\left(\mathbf{P}_{n}^{*} \notin \widehat{\mathbf{P}}_{n}+a_{n} U\right) \geqslant\left(\widehat{\mathbf{P}}_{n}\right)^{*}\left(\mathbf{P}_{n}^{*} \in \widehat{\mathbf{P}}_{n}+a_{n} \Omega_{0}\right) .
$$


The problem was reduced to finite dimensional.

For any finite set $H=\left\{h_{1}, \ldots, h_{m} ; h_{i} \in \Theta, 1 \leqslant i \leqslant m\right\}$ and any set $\Psi \subset \Lambda_{0 \Theta}$ denote

$$
\Psi_{H}=\left\{z=\left(z_{1}, \ldots, z_{m}\right): z_{i}=\mathbf{E}\left[h_{i}(X)\right], 1 \leqslant i \leqslant m\right\} .
$$

For all $i, j, 1 \leqslant j \leqslant l_{i}, 1 \leqslant i \leqslant m$, define signed measures $\mathbf{F}_{i j}$ having the densities $\frac{d \mathbf{F}_{i j}}{d \mathbf{P}}=f_{i j}-\mathbf{E}\left[f_{i j}(X)\right]$. Define linear spaces

$$
L=\left\{\mathbf{F}: \mathbf{F}=\sum_{i=1}^{k} \sum_{j=1}^{l_{i}} \lambda_{i j} \mathbf{F}_{i j}, \lambda_{i j} \in R^{1}, 1 \leqslant j \leqslant l_{i}, 1 \leqslant i \leqslant m\right\}
$$

and

$$
\widetilde{l}=\left\{f: f=\frac{d \mathbf{F}}{d \mathbf{P}}, \mathbf{F} \in L\right\} .
$$

Let $h_{1}, \ldots, h_{m_{1}}, h_{m_{1}+1}, \ldots, h_{m}$ be linear independent functions in $\widetilde{l}$ such that $\mathbf{E}\left[h_{i}^{2}(X)\right]=$ $2(\eta-\delta), 1 \leqslant i \leqslant m_{1}$, and $\mathbf{E}\left[h_{i}^{2}(X)\right]=0, m_{1}<i \leqslant m$. Define the sets $H=$ $\left\{h_{1}, \ldots, h_{m_{1}}\right\}$ and $H_{1}=\left\{h_{m_{1}+1}, \ldots, h_{m}\right\}$. We have

$$
\left(\mathfrak{c l}\left(\Omega_{0}\right)\right)_{H} \cap\left(\Gamma_{\eta-\delta}\right)_{H}=\emptyset .
$$

Denote $\Omega_{1}=\left\{\mathbf{G}: \mathbf{G} \in \mathfrak{c l}\left(\Omega_{0}\right), G_{H} \neq 0\right\}$ and $\Omega_{2}=\left\{\mathbf{G}: \mathbf{G} \in \mathfrak{c l}\left(\Omega_{0}\right), G_{H_{1}} \neq 0\right\}$.

It is clear that $\widehat{\mathbf{P}}_{n}\left(\mathbf{P}_{n}^{*} \notin \widehat{\mathbf{P}}_{n}+a_{n} \Omega_{1}\right)=0$ almost surely.

Therefore

$$
\begin{aligned}
\widehat{\mathbf{P}}_{n}\left(\mathbf{P}_{n}^{*} \in \widehat{\mathbf{P}}_{n}+a_{n} \Omega_{0}\right) \leqslant \widehat{\mathbf{P}}_{n}\left(\mathbf{P}_{n H}^{*} \in \widehat{\mathbf{P}}_{n H}+a_{n} \Omega_{1 H}\right) \leqslant \\
\widehat{\mathbf{P}}_{n}\left(\mathbf{P}_{n H}^{*} \notin \widehat{\mathbf{P}}_{n H}+a_{n}\left(\Gamma_{0, \eta-\delta}\right)_{H}\right) .
\end{aligned}
$$

Define the sets

$$
\widehat{\Gamma}_{0 c}=\left\{f: f=\frac{d F}{d \mathbf{P}}, F \in \Gamma_{0 c} \cap L\right\}, \quad c>0 .
$$

There is a finite number of functions $q_{1}, \ldots, q_{l} \in \widehat{\Gamma}_{0, \eta-2 \delta}$, such that

$$
\mathbf{E}\left[q_{i}(X)\right]=0, \quad \mathbf{E}\left[q_{i}^{2}(X)\right]=2(\eta-2 \delta), \quad 1 \leqslant i \leqslant l
$$

and

where

$$
\widehat{\Gamma}_{0, \eta-2 \delta} \cap L \subset \cap_{i=1}^{l} V\left(q_{i}\right) \cap L \subset \widehat{\Gamma}_{0, \eta-\delta} \cap L,
$$

Denote

$$
V_{i}=V\left(q_{i}\right)=\left\{\mathbf{G}:\left|\int q_{i} d \mathbf{G}\right|<2(\eta-2 \delta), \mathbf{G} \in \Lambda_{0 \Theta}\right\} .
$$

$$
V=\bigcap_{i=1}^{k} V_{i}
$$

Since $\widehat{\Gamma}_{0, \eta-\delta} \subset U \cap L$, then $V \subset U$. Therefore

$$
\Omega_{1} \subset W=\Lambda_{0 \Theta} \backslash V .
$$

Therefore it suffices to estimate the right-hand side

$$
\log \left(\widehat{\mathbf{P}}_{n}\right)^{*}\left(\mathbf{P}_{k_{n}}^{*} \in \widehat{\mathbf{P}}_{n}+a_{n} \Omega_{1}\right) \leqslant \log \widehat{\mathbf{P}}_{n}\left(\mathbf{P}_{k_{n}}^{*} \in \widehat{\mathbf{P}}_{n}+a_{n} W\right) .
$$

We have

$$
\begin{array}{r}
\widehat{\mathbf{P}}_{n}\left(\mathbf{P}_{k_{n}}^{*} \in \widehat{\mathbf{P}}_{n}+a_{n} W\right) \leqslant \sum_{i=1}^{k} \widehat{\mathbf{P}}_{n}\left(\mathbf{P}_{k_{n}}^{*} \notin \widehat{\mathbf{P}}_{n}+a_{n} U_{i}\right) \\
=\sum_{i=1}^{k} \widehat{\mathbf{P}}_{n}\left(\int q_{i} d\left(\mathbf{P}_{k_{n}}^{*}-\widehat{\mathbf{P}}_{n}\right)-2 a_{n}(\eta-2 \delta)>0\right) .
\end{array}
$$


Therefore it suffices to prove that, for each $f \in \Theta, \mathbf{E}[f(X)]=0, \mathbf{E}\left[f^{2}(X)\right]=\eta-2 \delta$ and $n>n_{0}(\epsilon, f)$, we have

$$
\begin{aligned}
& \left(k_{n} a_{n}^{2}\right)^{-1} \log \widehat{\mathbf{P}}_{n}\left(\int f d\left(\mathbf{P}_{k_{n}}^{*}-\widehat{\mathbf{P}}_{n}\right)>2 a_{n}(\eta-2 \delta)\right) \\
& \leqslant-2 \frac{(\eta-2 \delta)^{2}}{\operatorname{Var}\left[f\left(X_{1}\right)\right]}(1-\epsilon)=-2(\eta-2 \delta)(1-\epsilon)
\end{aligned}
$$

with probability $\kappa_{n}(\epsilon, U(f, q))$.

Denote $s^{2} \doteq s_{f}^{2} \doteq s_{n}^{2}=\frac{1}{n} \sum_{i=1}^{n} f^{2}\left(X_{i}\right)-\bar{f}^{2}$, where $\bar{f}=\frac{1}{n} \sum_{i=1}^{n} f\left(X_{i}\right)$. We put $\gamma=\frac{\sqrt{2} s \epsilon}{324 \sigma}$ where $\sigma^{2}=\operatorname{Var}\left[f\left(X_{1}\right)\right]=\eta-2 \delta$.

By Theorem 28 in [23, Ch. 4], we get $\mathbf{P}\left(\left|s_{n}^{2}-\sigma^{2}\right|>\epsilon\right)<\beta_{2 n}(f)$ where $\beta_{2 n}(f)=$ $C_{1}(f, \epsilon) n^{1-t / 2}$. Therefore, for the proof of (5.3), we can suppose that

$$
\left|s_{n}^{2}-\sigma^{2}\right|<\epsilon \text {. }
$$

Define the set of events

$$
A_{n f}=\left\{X_{1}, \ldots, X_{n}: \max _{1 \leqslant s \leqslant n}\left|f\left(X_{s}\right)\right|<\sigma \gamma a_{n}^{-1}\right\} .
$$

We have

$$
\mathbf{P}\left(A_{n f}\right) \geqslant 1-n \mathbf{P}\left(\left|f\left(X_{1}\right)\right|>\sigma \gamma a_{n}^{-1}\right)=1-n h\left(\frac{a_{n}}{\sigma \gamma}\right) \doteq 1-\beta_{2 n} .
$$

Note that, by (2.6),$n h\left(\frac{a_{n}}{\sigma \gamma}\right) \rightarrow 0$ as $n \rightarrow \infty$. Therefore it suffices to prove (5.3) if $A_{n f}$ holds.

The further reasoning are based on a slightly simplified version of Theorem 3.2 in [26. This version is provided below.

Let $Y_{1 n}, \ldots, Y_{k_{n}, n}$ be independent identically distributed random variables having probability measure $\mathbf{P}_{n}, \mathbf{E}\left[Y_{1 n}\right]=0$, Var $\left[Y_{1 n}\right]=\sigma^{2},\left|Y_{i n}\right|<\sigma \gamma a_{n}^{-1}$. Denote

$$
S_{n}=\frac{1}{\sqrt{k_{n}} \sigma} \sum_{i=1}^{k_{n}} Y_{i n} .
$$

Suppose that

$$
a_{n}^{-2} z^{-2} \log \mathbf{E}\left[\exp \left\{z a_{n} \sigma^{-1} Y_{1 n}\right\}\right]<C \text { for all }|z|<\kappa
$$

and

$$
\omega=\frac{\sqrt{2} \kappa}{36 \max \{1, C\}}>1 .
$$

Denote $\Delta=\omega a_{n} k_{n}^{1 / 2}$.

5.1. Assume (5.5) and (5.6). Then we have

$$
\begin{aligned}
\mathbf{P}\left(S_{n}>\right. & \left.k_{n}^{1 / 2} a_{n}\right) \\
& =\left(1-\Phi\left(k_{n}^{1 / 2} a_{n}\right)\right) \exp \left\{L\left(k_{n}^{1 / 2} a_{n}\right)\right\}\left(1+\theta f_{1}\left(k_{n}^{1 / 2} a_{n}\right) \frac{k_{n}^{1 / 2} a_{n}+1}{\Delta}\right),
\end{aligned}
$$

where

and

$$
f_{1}\left(k_{n}^{1 / 2} a_{n}\right)=\frac{60\left(1+10 \Delta^{2} \exp \left\{-\left(1-\omega^{-1}\right) \sqrt{\Delta}\right\}\right)}{1-\omega^{-1}}
$$

$$
-\frac{k_{n} a_{n}^{2}}{3 \omega}<L\left(k_{n}^{1 / 2} a_{n}\right)<\frac{k_{n} a_{n}^{2}}{2} \frac{1}{1+\omega} .
$$


Note that, if $\omega>16$ and $a_{n} k_{n}^{1 / 2}>100$, then we have

$$
\left|\theta_{1} f_{1}\left(k_{n}^{1 / 2} a_{n}\right)\right| \frac{k_{n}^{1 / 2} a_{n}+1}{\Delta}<6 .
$$

If $|z|<\kappa$ and $\left|f\left(X_{i}\right)\right|<\sigma \gamma a_{n}^{-1}, 1 \leqslant i \leqslant n$, then

$$
\begin{aligned}
& \log \mathbf{E}_{\widehat{\mathbf{P}}_{n}}\left\{\exp \left\{z a_{n}\left(f\left(X_{1}^{*}\right)-\bar{f}\right) / s\right\}\right\} \\
& =\log \left[\frac{1}{n} \sum_{l=1}^{n} \exp \left\{z a_{n}\left(f\left(X_{i}\right)-\bar{f}\right) / s\right\}\right] \\
& =\log \left(1+\frac{z^{2} a_{n}^{2}}{2}+\frac{\theta^{3} z^{3} a_{n}^{3} s^{-3}}{6 n} \sum_{i=1}^{n}\left(f\left(X_{i}\right)-\bar{f}\right)^{3} \exp \left\{\theta z a_{n}\left(f\left(X_{i}\right)-\bar{f}\right) / s\right\}\right) \\
& \doteq \tau_{n}
\end{aligned}
$$

where $0<\theta<1$.

Since

$$
\exp \left\{\theta z a_{n}\left(f\left(X_{1}\right)-\bar{f}\right) / s\right\}<\exp \left\{2 \gamma \kappa \theta \sigma s^{-1}\right\} \doteq R
$$

using $\log (1+x)<x, x>0$, we get

$$
\tau_{n}<\log \left(1+\frac{z^{2} a_{n}^{2}}{2}\left(1+\gamma \kappa \sigma R s^{-1}\right)\right)<\frac{z^{2} a_{n}^{2}}{2}\left(1+\gamma \kappa \sigma R s^{-1}\right)=z^{2} a_{n}^{2} D
$$

where $D=\frac{1+\gamma \kappa R \sigma s^{-1}}{2}$.

If

$$
\kappa=\frac{s}{2 \gamma \sigma}
$$

then $R<3$ and $D<2$. Therefore

$$
\omega>\frac{9}{2 \epsilon}, \quad L\left(k_{n}^{1 / 2} a_{n}\right) \leqslant \frac{k_{n}^{1 / 2} a_{n}^{2}}{2} \frac{\epsilon}{9 / 2+\epsilon} .
$$

Hence, by (5.7) and (5.9), we get

$$
\begin{aligned}
\left(k_{n} a_{n}^{2}\right)^{-1} \log \widehat{\mathbf{P}}_{n}\left(\int f d\left(\mathbf{P}_{k_{n}}^{*}-\widehat{\mathbf{P}}_{n}\right)>2 a_{n}(\eta-2 \delta)\right) \\
\leqslant-\frac{1}{2} s^{-2}(\eta-2 \delta)^{2}\left(1-\frac{\epsilon}{9 / 2+\epsilon}\right) \\
\quad+\left(\log 7-\frac{1}{2} \log \left(2 \pi s^{-2}(1+\epsilon)\right)\right)\left(k_{n} a_{n}^{2}\right)^{-1} \\
\leqslant-\frac{1}{2} s^{-2}(\eta-2 \delta)^{2}\left(1-\frac{\epsilon}{2}\right)+C\left(k_{n} a_{n}^{2}\right)^{-1} \\
=-\frac{1}{2} s^{-2}(\eta-2 \delta)^{2}\left(1-\frac{\epsilon}{2}\right)+C\left(k_{n} a_{n}^{2}\right)^{-1} \\
\leqslant-\frac{1}{2} s^{-2}(\eta-2 \delta)^{2}\left(1-\frac{\epsilon}{2}\right)+C\left(k_{n} a_{n}^{2}\right)^{-1} .
\end{aligned}
$$

This implies (5.3), if (5.4) and $\left|f\left(X_{i}\right)\right|<\sigma \gamma a_{n}^{-1}, 1 \leqslant i \leqslant n$ hold. This completes the proof of (2.8).

If $\rho_{0}^{2}\left(\mathfrak{c l}\left(\Omega_{0}\right), \mathbf{P}\right)=\infty$, we put $\eta=L$. After that it suffices to implement the same reasoning as in the proof of (2.8).

The proof of lower bound (2.7) is based on standard reasoning (see [8, 10, 24] and references therein) and estimates of Theorem 5.1. For any $\delta>0$ there is open set $U=U\left(f_{1}, \ldots, f_{l}, \mathbf{G}, \gamma\right)$ such that $U \subset \mathfrak{i n t}\left(\Omega_{0}\right)$ and $\rho_{0}^{2}(U, \mathbf{P})<\eta+\delta$, $\rho_{0}^{2}(\mathbf{G}, \mathbf{P})<\eta+\delta$. Therefore it suffices to find lower bound for the asymptotic

$$
\left(k_{n} a_{n}^{2}\right)^{-1} \log \widehat{\mathbf{P}}_{n}\left(\mathbf{P}_{k}^{*} \in \widehat{\mathbf{P}}_{n}+a_{n} U\right) .
$$


Arguing similarly to the proof of upper bound, we can suppose that the signed measure $\mathbf{G}$ has the density $g=\frac{d \mathbf{G}}{d \mathbf{P}}=\sum_{i=1}^{l} \lambda_{i} f_{i}, f_{i} \in \Theta$. Thus the problem is finite dimensional.

We fix $\lambda, 0<\lambda<1$ such that $\lambda \mathbf{G} \in U$. Note that $\lambda$ may be defined arbitrary from some vicinity of 1 . Define the set $U_{1}=U \cap U\left(g, \mathbf{G},(1-\lambda)^{2}\|g\|^{2}\right)$. It is clear that we can choose $\lambda$ such that $\rho_{0}^{2}\left(U_{1}, \mathbf{P}\right) \leqslant \frac{1}{2} \lambda^{2}\|g\|^{2}$.

5.2. There is simplex $\widetilde{U} \subset U_{1}$ bounded the hyperplane

$$
\Pi=\left\{\mathbf{R}: \int g d \mathbf{R}=\lambda^{2}\|g\|^{2}, \mathbf{R} \in \Lambda_{0 \Theta}\right\}
$$

and the hyperplanes

$$
\Pi_{i}=\left\{\mathbf{R}: \int g_{i} d \mathbf{R}=c_{i}, \mathbf{R} \in \Lambda_{0 \Theta}\right\},
$$

with $g_{i} \in \Theta, 1 \leqslant i \leqslant l$ such that $\rho_{0}^{2}\left(\Pi_{i}, \mathbf{P}\right) \geqslant \lambda^{2}\|g\|^{2}>\rho_{0}^{2}(\Pi, \mathbf{P})$.

The proof of Lemma 5.2 will be given later. Let Lemma 5.2 be valid. Suppose that $A_{b f}$ holds with $f=g$ and $f=g_{i}, 1 \leqslant i \leqslant l$. Then, implementing Theorem 5.1 and Lemma 5.2, we get

$$
\begin{aligned}
& \widehat{\mathbf{P}}_{n}\left(\mathbf{P}_{k_{n}}^{*} \in \widehat{\mathbf{P}}_{n}+a_{n} U_{1}\right) \geqslant \widehat{\mathbf{P}}_{n}\left(\mathbf{P}_{k_{n}}^{*} \in \widehat{\mathbf{P}}_{n}+a_{n} \widetilde{U}\right) \\
& \geqslant \widehat{\mathbf{P}}_{n}\left(\int g d\left(\mathbf{P}_{k_{n}}^{*}-\widehat{\mathbf{P}}_{n}\right)>\lambda^{2}\|g\|^{2} a_{n}\right) \\
& \quad-\sum_{i=1}^{l} \widehat{\mathbf{P}}_{n}\left(\int g_{i}\left(d \mathbf{P}_{k_{n}}^{*}-\widehat{\mathbf{P}}_{n}\right)>a_{n} c_{i}\right) \\
& \geqslant \widehat{\mathbf{P}}_{n}\left(\int g d\left(\mathbf{P}_{k_{n}}^{*}-\widehat{\mathbf{P}}_{n}\right)>\lambda^{2}\|g\|^{2} a_{n}\right) \\
& -\sum_{i=1}^{l} \exp \left\{-\rho_{0}^{2}\left(\Pi_{i}, \mathbf{P}\right) a_{n}^{2} k_{n}\left(1+\epsilon_{n}\right)\right\}
\end{aligned}
$$

where $\epsilon_{n} \rightarrow 0$ as $n \rightarrow \infty$.

Thus, it remains to implement Theorem 5.1 to the first addendum of right-hand side of (5.10).

By (5.7) and (5.8), we get

$$
\begin{aligned}
& \left.\left(a_{n}^{2} k_{n}\right)^{-1} \log \widehat{\mathbf{P}}_{n}\left(\int g d \mathbf{P}_{k_{n}}^{*}-\widehat{\mathbf{P}}_{n}\right)>a_{n} \lambda^{2}\|g\|^{2}\right) \\
& \geqslant-\frac{1}{2} \lambda^{2}\|g\|^{2}\left(1+\frac{1}{3 \omega}\right)+c\left(k_{n} a_{n}^{2}\right)^{-1} \\
& =-\frac{1}{2} \lambda^{2}\|g\|^{2}\left(1+\frac{s}{9 \sigma} \epsilon\right)+c\left(k_{n} a_{n}^{2}\right)^{-1} .
\end{aligned}
$$

This implies the lower bound.

Proof of Lemma 5.2. The problem is reduced to the following. Let we be given a parallelepiped $U_{1}$ in $R^{l+1}$ and $0 \notin U_{1}$. Let the point $u$ lies on the face $\Pi$ of parallelepiped $U_{1}$ and $|u|=\rho\left(0, U_{1}\right)=\inf _{x \in U_{1}}|x|$. One needs to point out simplex $V \subset U_{1}$ such that $\Pi \cap V$ is the face of $V, u \in \Pi \cap V$ and, for any hyperplane $\Pi_{1}$ passing through another face of $\mathrm{V}$, it holds $\rho\left(0, \Pi_{1}\right)>\rho(0, u)$. Let the distance of $u$ from any face other than $\Pi$ exceeds $r_{0}$. A simple trigonometric reasoning shows that the simplex $V$ can be defined as follows. We take the vertex $v=\left(1+\frac{1}{2} r^{2}\right) u$ of $V$ where $r<<r_{0}$ and all other vertices $v_{i}, 1 \leqslant i \leqslant l$ belong $\Pi$ and $\left|v_{i}-u\right|=r$. 
For the proof of this statement it suffices to consider the case $l=1$. Let us draw through $v$ the line $L$ intersecting the line $\Pi$ at the point $w$ and such that $w$ is orthogonal to $L$. Then $|u-v|=|w-u|^{2}|u|^{-1}(1+o(1))$. Therefore, if the line $L_{1}, v \in L_{1}$ intersect $\Pi$ at the point $z=c|w-u|^{2}|u|^{-1}, c<1$, then $\rho\left(0, L_{1}\right)>|u|$.

Proof of Theorem 2.1. The reasoning are based on estimates of Theorem 2.2.

We begin with the proof of upper bound (2.5) in the case of $\tau_{\Theta_{2 h}}$-topology. Suppose that $\rho_{0}^{2}\left(\mathfrak{c l}\left(\Omega_{0}\right), \mathbf{P}\right)<\infty$. If $\rho_{0}^{2}\left(\mathfrak{c l}\left(\Omega_{0}\right), \mathbf{P}\right)=\infty$, the reasoning are similar. It suffices to prove that, for any $\epsilon>0$, there holds

$$
\left(k_{n} a_{n}^{2}\right)^{-1} \log \left(\widehat{\mathbf{P}}_{n}\right)^{*}\left(\mathbf{P}_{k_{n}}^{*} \in \widehat{\mathbf{P}}_{n}+a_{n} \Omega_{0}\right) \leqslant-\rho_{0}^{2}\left(\mathfrak{c l}\left(\Omega_{0}\right), \mathbf{P}\right)+\epsilon \quad a . s^{*} .
$$

By Strong Law of Large Numbers and (2.2), for any $f \in \Theta$ there holds

$$
s_{n}^{2}(f) \rightarrow \sigma^{2}(f) \quad \text { a.s. }
$$

with $\sigma^{2}(f)<\infty$.

By (2.3) and (2.1), for any $\delta>0$ we have

$$
\begin{aligned}
& \mathbf{P}\left(\max _{i \geqslant l} a_{i}\left|f\left(X_{i}\right)\right| \leqslant \delta\right)=\prod_{i=l}^{\infty}\left(1-\mathbf{P}\left(\left|f\left(X_{i}\right)\right|>\delta a_{s}^{-1}\right)\right) \\
& \geqslant \prod_{i=l}^{\infty}\left(1-h\left(a_{i} / \delta\right)\right) \geqslant \exp \left\{-\sum_{i=l}^{\infty} h\left(a_{i} / \delta\right)\right\}=1+o(1)
\end{aligned}
$$

as $l \rightarrow \infty$.

For each $k$

$$
\mathbf{P}\left(\max _{1 \leqslant i \leqslant k} a_{n}\left|f\left(X_{i}\right)\right|>\delta\right)=o(1) \quad \text { as } n \rightarrow \infty .
$$

Note that $\max _{i \geqslant k} a_{i}\left|f\left(X_{i}\right)\right|<\delta$ implies $\max _{k \leqslant i \leqslant n}\left|f\left(X_{i}\right)\right|<\delta a_{n}^{-1}$. Therefore, by (5.12) and (5.13), we get

$$
\max _{1 \leqslant s \leqslant n}\left|f\left(X_{s}\right)\right|<\delta a_{n}^{-1} \quad \text { a.s. }
$$

Using (5.11) and (5.14), we can implement the same technique for the proof of (5.3), as in the proof of (2.7) in Theorem 2.2. This completes the proof of (2.5).

For the proof of (2.5) in the case of $\tau_{\Theta_{t}}$-topology, it suffices to show that, for any $\delta>0$, there holds

$$
I_{k} \doteq \mathbf{P}\left(\max _{i>k} a_{i}\left|f\left(X_{i}\right)\right|>\delta\right)=o(1) \quad \text { as } k \rightarrow \infty .
$$

We have

$$
I_{k} \leqslant \sum_{i=k}^{\infty} \mathbf{P}\left(f\left(X_{i}\right)>\delta a_{i}^{-1}\right)=\sum_{i=k+1}^{\infty}(i-k) \mathbf{P}\left(\delta a_{i-1}^{-1}<\left|f\left(X_{1}\right)\right| \leqslant \delta a_{i}^{-1}\right) \doteq J_{k} .
$$

Define the function $u(x)=\delta a_{i-1}^{-1}+\delta\left(x-a_{i-1}^{-1}\right)$, if $x \in\left[a_{i-1}^{-1}, a_{i}^{-1}\right)$. Define the inverse function $v(y)=\inf \left\{t: u(t)=y, t \in R^{1}\right\}$. Define the distribution function $F(x)=\mathbf{P}\left(\left|f\left(X_{1}\right)\right|<x\right), x \in R_{+}^{1}$.

Then

$$
J_{k} \leqslant 2 \int_{a_{k}^{-1}}^{\infty} v(x) d F(x) \leqslant 2 \int_{a_{k}^{-1}}^{\infty} x^{t} d F(x)=o(1) \quad k \rightarrow \infty .
$$

This implies (5.15).

The proof of lower bound (2.4) is based on similar reasoning and is omitted. 


\section{Proof of Theorem 3.1}

For all $r>0$ define the set

$$
\Gamma_{r}=\left\{\overline{\mathbf{G}} \in \Lambda_{0}^{2}: \rho_{0 b}^{2}(\overline{\mathbf{G}}: \mathbf{P}) \leqslant r\right\} .
$$

6.1. Let 3.4 hold. Then

(i) $\Gamma_{r} \subset \Lambda_{0 \Phi}^{2}$,

(ii) the set $\Gamma_{r}$ is $\tau_{\Phi}$-compact and sequentially $\tau_{\Phi}$-compact set in $\Lambda_{0 \Phi}^{2}$,

(iii) the $\tau$ and $\tau_{\Phi}$-topologies coincide in $\Gamma_{r}$.

The proof of Lemma 6.1 is akin to the proof of Lemma 5.1 and is omitted.

The same reasoning as in the proof of Lemma 6.1 can be repeated in the case of $\tau_{\Psi}$-topology. Thus the sets $\Gamma_{0 r}$ are $\tau_{\Psi}$-compacts as well.

In Lemmas 4.2-4.5 given below we suppose that the assumptions of Theorem 3.1 are satisfied.

For all $u, v \in R^{k}$ denote $u^{\prime} v$ the inner product of vectors $u$ and $v$. For all $f \in \Phi$ and all $\mathbf{G} \in \Lambda_{0 \Phi}$ denote $\langle f, \mathbf{G}\rangle=\int f d \mathbf{G}$.

Let $f_{1}, \ldots, f_{k_{1}}, g_{1}, \ldots, g_{k_{2}} \in \Phi$ and $\mathbf{G} \in \Lambda_{0 \Phi}$. Let $\mathbf{E}\left[f_{i}(X)\right]=0, \mathbf{E}\left[g_{j}(X)\right]=0$ for $1 \leqslant i \leqslant k_{1}, 1 \leqslant j \leqslant k_{2}$. Define covariance matrices

$$
R_{f}=\left\{\mathbf{E}\left[f_{i}(X) f_{j}(X)\right]\right\}_{i, j=1}^{k_{1}} \text { and } R_{g}=\left\{\mathbf{E}\left[g_{i}(X) g_{j}(X)\right]\right\}_{i, j=1}^{k_{2}} .
$$

Denote $\vec{f}=\left\{f_{i}\right\}_{i=1}^{k_{1}}, \vec{g}=\left\{g_{i}\right\}_{i=1}^{k_{2}}$ and $\bar{g}_{i}=\frac{1}{n} \sum_{l=1}^{n} g_{i}\left(X_{l}\right), 1 \leqslant i \leqslant k_{2}$.

By Dawson-Gartner Theorem (see 10 Theorem 4.6.9 and 21]), Theorem 3.1 follows from Lemma 6.2 given below. Note that the de Acosta 2] approach (see section 5) also allows to deduce Theorem 3.1 from Lemma 6.2

6.2. For random vectors

$$
\begin{aligned}
\vec{U}_{n}(\vec{X})=\left(\frac{1}{n} \sum_{i=1}^{n} f_{1}\left(X_{i}\right), \ldots, \frac{1}{n} \sum_{i=1}^{n} f_{k_{1}}\left(X_{i}\right),\right. & \\
& \left.\frac{1}{n} \sum_{i=1}^{n} g_{1}\left(X_{i}^{*}\right)-\bar{g}_{1}, \ldots, \frac{1}{n} \sum_{i=1}^{n} g_{k_{2}}\left(X_{i}^{*}\right)-\bar{g}_{k_{2}}\right)
\end{aligned}
$$

$L D P$ holds, that is, for every $\Omega \subset R^{k_{1}+k_{2}}$, there holds

$$
\liminf _{n \rightarrow \infty}\left(n b_{n}^{2}\right)^{-1} \log \mathbf{P}_{n}\left(\vec{U}_{n}(\vec{X}) \in b_{n} \Omega\right) \geqslant-\inf _{x \in \operatorname{int}(\Omega)} x^{\prime} I_{f, g} x
$$

and

$$
\limsup _{n \rightarrow \infty}\left(n b_{n}^{2}\right)^{-1} \log \mathbf{P}_{n}\left(\vec{U}_{n}(\vec{X}) \in b_{n} \Omega\right) \leqslant-\inf _{x \in \operatorname{cl}(\Omega)} x^{\prime} I_{f, g} x
$$

where, for each $x=(y, z) \in R^{k_{1}+k_{2}}, y \in R^{k_{1}}$ and $z \in R^{k_{2}}$,

$$
x^{\prime} I_{f, g} x=\sup _{t \in R^{k_{1}, s \in R^{k_{2}}}}\left(t^{\prime} y+s^{\prime} z-\left\langle t^{\prime} f, H\right\rangle-\frac{1}{2} t^{\prime} R_{f} t-\frac{1}{2} s^{\prime} R_{g} s\right) .
$$

Note that, if there is $R_{f}^{-1}$ and $R_{g}^{-1}$, then

$$
x^{\prime} I_{f g} x=\frac{1}{2}(y-\langle f, H\rangle)^{\prime} R_{f}^{-1}(y-\langle f, H\rangle)+\frac{1}{2} z^{\prime} R_{g}^{-1} z .
$$

Lemma 6.2 follows from Lemmas 6.3 and 6.4 given below.

6.3. There hold

$$
\lim _{n \rightarrow \infty}\left(n b_{n}^{2}\right)^{-1} \log \mathbf{P}_{n}\left(\max _{1 \leqslant i \leqslant k_{1}} \max _{1 \leqslant l \leqslant n}\left|f_{i}\left(X_{l}\right)\right|>b_{n}^{-1}\right)=-\infty
$$

and

$$
\lim _{n \rightarrow \infty}\left(n b_{n}^{2}\right)^{-1} \log \mathbf{P}_{n}\left(\max _{1 \leqslant i \leqslant k_{2}} \max _{1 \leqslant l \leqslant n}\left|g_{i}\left(X_{l}^{*}\right)\right|>b_{n}^{-1}\right)=-\infty .
$$


Proof. We have

$$
\begin{aligned}
& \mathbf{P}_{n}\left(\max _{1 \leqslant i \leqslant k_{1}} \max _{1 \leqslant l \leqslant n}\left|f_{i}\left(X_{l}\right)\right|>b_{n}^{-1}\right) \leqslant n \sum_{i=1}^{k_{1}} \mathbf{P}_{n}\left(\left|f_{i}\left(X_{1}\right)\right|>b_{n}^{-1}\right) \\
\leqslant & n \sum_{i=1}^{k_{1}} \mathbf{P}\left(\left|f_{i}\left(X_{1}\right)\right|>b_{n}^{-1}\right)+n b_{n} \sum_{i=1}^{k_{1}} \int \chi\left(\left|f_{i}\left(X_{1}\right)\right|>b_{n}^{-1}\right) d\left|\mathbf{H}_{n}\right| .
\end{aligned}
$$

By (3.1) and B1, this implies (6.3).

Since $g_{1}, \ldots, g_{k_{2}} \in \Phi$, the same statement holds for these functions as well and we get

$$
\mathbf{P}_{n}\left(\max _{1 \leqslant i \leqslant k_{2}} \max _{1 \leqslant j \leqslant n}\left|g_{i}\left(X_{j}\right)\right|>b_{n}^{-1}\right)=O\left(\exp \left\{-C n b_{n}^{2}\right\}\right)
$$

for any $C>0$. This implies (6.4).

For each $h \in \Phi$ denote $h_{n}(x)=h(x) \chi\left(|h(x)|<b_{n}^{-1}\right)$. Denote $\vec{f}_{n}=\left\{f_{i n}\right\}_{i=1}^{k_{1}}$ $\vec{g}_{n}=\left\{g_{i n}\right\}_{i=1}^{k}$. Define random vector

$$
\begin{aligned}
\widetilde{U}_{n}(\vec{X})=\left(\frac{1}{n} \sum_{i=1}^{n} f_{1 n}\left(X_{i}\right), \ldots,\right. & \frac{1}{n} \sum_{i=1}^{n} f_{k_{1} n}\left(X_{i}\right) \\
& \left.\frac{1}{n} \sum_{i=1}^{n} g_{1 n}\left(X_{i}^{*}\right)-\bar{g}_{1 n}, \ldots, \frac{1}{n} \sum_{i=1}^{n} g_{k_{2} n}\left(X_{i}^{*}\right)-\bar{g}_{k_{2} n}\right)
\end{aligned}
$$

with $\bar{g}_{\text {in }}=\frac{1}{n} \sum_{l=1}^{n} g_{\text {in }}\left(X_{l}\right), 1 \leqslant i \leqslant k_{2}$. Define the sets of events

$$
\begin{aligned}
& W_{n}=\left\{X_{1}, \ldots, X_{n}: \max _{1 \leqslant i \leqslant k_{1}} \max _{1 \leqslant j \leqslant n}\left|f_{i}\left(X_{j}\right)\right|<b_{n}^{-1},\right. \\
& \left.\max _{1 \leqslant i \leqslant k_{2}} \max _{1 \leqslant j \leqslant n}\left|g_{i}\left(X_{j}\right)\right|<b_{n}^{-1}\right\} .
\end{aligned}
$$

Denote $\bar{W}_{n}$ the complement of $W_{n}$. By Lemma 6.2 we get

$$
\begin{aligned}
& \mathbf{P}_{n}\left(\vec{U}_{n}(\vec{X}) \in b_{n} \Omega\right) \leqslant \mathbf{P}_{n}\left(\vec{U}_{n}(\vec{X}) \in b_{n} \Omega \mid \bar{W}_{n}\right) \mathbf{P}\left(\bar{W}_{n}\right)+\mathbf{P}\left(W_{n}\right) \\
& <\mathbf{P}_{n}\left(\vec{U}_{n}(\vec{X}) \in b_{n} \Omega \mid \bar{W}_{n}\right) \exp \left\{o\left(n b_{n}^{2}\right)\right\}+\exp \left\{-C n b_{n}^{2}(1+o(1))\right\}
\end{aligned}
$$

and

$$
\begin{aligned}
\mathbf{P}_{n}\left(\vec{U}_{n}(\vec{X}) \in b_{n} \Omega\right) \geqslant \mathbf{P}_{n}\left(\vec{U}_{n}(\vec{X}) \in b_{n} \Omega \mid \bar{W}_{n}\right) \mathbf{P}\left(\bar{W}_{n}\right) & \\
& >\mathbf{P}_{n}\left(\vec{U}_{n}(\vec{X}) \in b_{n} \Omega \mid \bar{W}_{n}\right) \exp \left\{o\left(n b_{n}^{2}\right)\right\}
\end{aligned}
$$

where the constant $C$ in (6.5) can be choosed arbitrary.

Thus, Lemma 6.2 follows from Lemma 6.4 given below.

6.4. For random vectors $\widetilde{U}_{n}(\vec{X})$ LDP holds, that is, (6.1) and (6.2) are valid for $\vec{U}_{n}(\vec{X})=\widetilde{U}_{n}(\vec{X})$.

By Gartner-Ellis Theorem (see [10, Lemma 6.4), Lemma 6.4 follows from Lemma 6.5 given below.

6.5. Let $f_{i} \in \Phi, g_{j} \in \Phi$ for all $1 \leqslant i \leqslant k_{1}, 1 \leqslant j \leqslant k_{2}$. Then

$$
\begin{aligned}
\lim _{n \rightarrow \infty}\left(n b_{n}^{2}\right)^{-1} \log \mathbf{E}_{n}\left[\operatorname { e x p } \left\{b_{n} \sum_{l=1}^{n} t^{\prime} \vec{f}_{n}\left(X_{l}\right)\right.\right. & \left.\left.+b_{n} \sum_{l=1}^{n} s^{\prime}\left(\vec{g}_{n}\left(X_{l}^{*}\right)-\bar{g}_{n}\right)\right\}\right] \\
& =\left\langle t^{\prime} \vec{f}, H\right\rangle-\frac{1}{2} t^{\prime} R_{f} t-\frac{1}{2} s^{\prime} R_{g} s
\end{aligned}
$$

with $\bar{g}_{n}=\left(\bar{g}_{1 n}, \ldots, \bar{g}_{k_{2} n}\right)$. 
Proof. We begin with the proof of upper bound in (6.6). We have

$$
\begin{aligned}
I_{n}= & \mathbf{E}_{n}\left[\exp \left\{b_{n} \sum_{l=1}^{n} t^{\prime} \vec{f}_{n}\left(X_{l}\right)+b_{n} \sum_{l=1}^{n} s^{\prime}\left(\vec{g}_{n}\left(X_{l}^{*}\right)-\bar{g}_{n}\right)\right\}\right] \\
= & \mathbf{E}_{n}\left[\exp \left\{b_{n} \sum_{l=1}^{n} t^{\prime} \vec{f}_{n}\left(X_{l}\right)\right\} \prod_{l=1}^{n} \mathbf{E}_{\widehat{\mathbf{P}}_{n}}\left[\exp \left\{s^{\prime}\left(\vec{g}_{n}\left(X_{l}^{*}\right)-\bar{g}_{n}\right)\right\}\right]\right] \\
= & \mathbf{E}_{n}\left[\exp \left\{b_{n} \sum_{l=1}^{n} t^{\prime} \vec{f}_{n}\left(X_{l}\right)\right\}\left(\frac{1}{n} \sum_{l=1}^{n} \exp \left\{b_{n} s^{\prime}\left(\vec{g}_{n}\left(X_{l}\right)-\bar{g}_{n}\right)\right\}\right)^{n}\right] \\
\leqslant & \mathbf{E}_{n}\left[\operatorname { e x p } \{ b _ { n } \sum _ { l = 1 } ^ { n } t ^ { \prime } \vec { f } _ { n } ( X _ { l } ) \} \left(1+\frac{b_{n}^{2}}{2 n} \sum_{l=1}^{n}\left(s^{\prime}\left(\vec{g}_{n}\left(X_{l}\right)-\bar{g}_{n}\right)\right)^{2}\right.\right. \\
& \left.\left.+C\left(s, k_{2}\right) \frac{b_{n}^{3}}{6 n} \sum_{l=1}^{n}\left|s^{\prime}\left(\vec{g}_{n}\left(X_{l}\right)-\bar{g}_{n}\right)\right|^{3}\right)^{n}\right] \\
\leqslant & \mathbf{E}_{n}\left[\operatorname { e x p } \left\{b_{n} \sum_{l=1}^{n} t^{\prime} \vec{f}_{n}\left(X_{l}\right)+\frac{b_{n}^{2}}{2} \sum_{l=1}^{n}\left(s^{\prime}\left(\vec{g}_{n}\left(X_{l}\right)-\bar{g}_{n}\right)\right)^{2}\right.\right. \\
& \left.\left.+C\left(s, k_{2}\right) b_{n}^{3} \sum_{l=1}^{n}\left|s^{\prime}\left(\vec{g}_{n}\left(X_{l}\right)-\bar{g}_{n}\right)\right|^{3}\right\}\right] \doteq I_{1 n} .
\end{aligned}
$$

The first inequality in (6.7) follows from Taylor formula and

$$
\left|s^{\prime}\left(\overrightarrow{g_{n}}(x)-\bar{g}_{n}\right)\right| \leqslant|s|\left|\overrightarrow{g_{n}}(x)-\bar{g}_{n}\right|<|s| 2 k_{2}^{1 / 2} b_{n}^{-1} .
$$

Denote $\phi_{n}\left(X_{l}\right)=s^{\prime}\left(\vec{g}_{n}\left(X_{l}\right)-\mathbf{E}_{n}\left[\vec{g}_{n}\left(X_{1}\right)\right]\right)$ with $1 \leqslant l \leqslant n$.

By straightforward calculations, we get

$$
\sum_{l=1}^{n}\left(s^{\prime}\left(\vec{g}_{n}\left(X_{l}\right)-\bar{g}_{n}\right)\right)^{2}=\sum_{l=1}^{n} \phi_{n}^{2}\left(X_{l}\right)-n\left(s^{\prime} \bar{g}_{n}-\mathbf{E}_{n}\left[s^{\prime} \vec{g}_{n}\left(X_{1}\right)\right]\right)^{2} .
$$

We have

$$
\begin{aligned}
\sum_{l=1}^{n} \mid s^{\prime}\left(\vec{g}_{n}\left(X_{l}\right)\right. & \left.-\bar{g}_{n}\right)\left.\right|^{3} \\
& \leqslant \sum_{l=1}^{n}\left|\phi_{n}\left(X_{l}\right)\right|^{3}+8 n\left|s^{\prime}\left(\bar{g}_{n}-\mathbf{E}_{n}\left[\vec{g}_{n}\left(X_{1}\right)\right]\right)\right|^{3} \doteq 8 V_{1}+8 n V_{2} .
\end{aligned}
$$

Since

$$
\begin{aligned}
& \left|s^{\prime}\left(\vec{g}_{n}\left(X_{1}\right)-\mathbf{E}\left[g_{n}\left(X_{1}\right)\right]\right)\right|^{3} \leqslant|s|^{3 / 2}\left|\vec{g}_{n}\left(X_{1}\right)-\mathbf{E}_{n}\left[g_{n}\left(X_{1}\right)\right]\right|^{3 / 2} \\
& =|s|^{3 / 2}\left(\sum_{j=1}^{k_{2}}\left(g_{j n}\left(X_{1}\right)-\mathbf{E}_{n}\left[g_{j n}\left(X_{1}\right)\right)^{2}\right)^{3 / 2}<8|s|^{3} k_{2}^{3 / 2} b_{n}^{-3},\right.
\end{aligned}
$$

then

$$
\begin{aligned}
& b_{n}^{3}\left|V_{1}\right|=b_{n}^{3} \sum_{l=1}^{n}\left|\phi_{n}\left(X_{l}\right)\right|^{3} \chi\left(\left|\phi_{n}\left(X_{l}\right)\right| \leqslant \epsilon b_{n}^{-1}|s|\right) \\
& +b_{n}^{3} \sum_{l=1}^{n}\left|\phi_{n}\left(X_{l}\right)\right|^{3} \chi\left(\left|\phi_{n}\left(X_{l}\right)\right| \geqslant \epsilon b_{n}^{-1}|s|\right) \\
& \leqslant \epsilon|s| b_{n}^{2} \sum_{l=1}^{n} \phi_{n}^{2}\left(X_{l}\right)+8|s|^{3} k_{2}^{3 / 2} \sum_{l=1}^{n} \chi\left(\left|\phi_{n}\left(X_{l}\right)\right| \geqslant \epsilon b_{n}^{-1}|s|\right) .
\end{aligned}
$$


Implementing Jensen's inequality, we get

$$
V_{2}=n^{-3}\left|\sum_{l=1}^{n} \phi_{n}\left(X_{l}\right)\right|^{3} \leqslant n^{-1} \sum_{l=1}^{n}\left|\phi_{n}\left(X_{l}\right)\right|^{3}=n^{-1} V_{1} .
$$

Using (6.8)- -6.12 , we get

$$
\begin{aligned}
I_{1 n} & \leqslant \mathbf{E}_{n}\left[\operatorname { e x p } \left\{b_{n} \sum_{l=1}^{n} t^{\prime} \vec{f}_{n}\left(X_{l}\right)+\frac{b_{n}^{2}}{2}\left(1-2 C\left(s, k_{2}\right) \epsilon_{n}\right) \sum_{l=1}^{n}\left(\phi_{n}^{2}\left(X_{l}\right)\right.\right.\right. \\
& \left.\left.\left.-\frac{b_{n}^{2}}{2 n}\left(\sum_{l=1}^{n} \phi_{n}\left(X_{l}\right)\right)^{2}+C\left(s, k_{2}\right)|s|^{3} \sum_{i=1}^{n} \chi\left(\left|\phi_{n}\left(X_{l}\right)\right| \geqslant \epsilon b_{n}^{-1}|s|\right)\right)\right\}\right] \\
& \doteq \mathbf{E}_{n}\left[W_{n}\right]
\end{aligned}
$$

where $\epsilon=\epsilon_{n} \rightarrow 0$ as $n \rightarrow \infty$. For all $r>0$, define the events

$$
A_{n}=A_{n r} \doteq\left\{X_{1}, \ldots, X_{n}: s^{\prime} \bar{g}_{n}-\mathbf{E}_{n}\left[s^{\prime} g_{n}\left(X_{1}\right)\right]<r b_{n}\right\} \text {. }
$$

Denote $\bar{A}_{n}$ the complement of $A_{n}$.

We have

$$
\widetilde{I}_{n}=\mathbf{E}_{n}\left[W_{n} \chi\left(A_{n}\right)\right]+\mathbf{E}_{n}\left[W_{n} \chi\left(\bar{A}_{n}\right)\right] \doteq U_{1 n}+U_{2 n} .
$$

Let $A_{n}$ holds. Then

$$
\frac{r^{2} b_{n}^{4}}{2 n}\left(\sum_{l=1}^{n} \phi_{n}\left(X_{l}\right)\right)^{2}=\frac{n b_{n}^{2}}{2}\left(s^{\prime} \bar{g}_{n}-\mathbf{E}_{n}\left[s^{\prime} \vec{g}_{n}(X)\right]\right)^{2}<\frac{n r^{2} b_{n}^{4}}{2} .
$$

Therefore we have

$$
\begin{aligned}
& \log \left[U_{1 n}\right] \leqslant \log \mathbf{E}_{n}\left[\operatorname { e x p } \left\{b_{n} \sum_{l=1}^{n} t^{\prime} \vec{f}_{n}\left(X_{l}\right)+\frac{b_{n}^{2}}{2} \sum_{l=1}^{n} \phi_{n}^{2}\left(X_{l}\right)\left(1+2 C\left(s, k_{2}\right) \epsilon\right)\right.\right. \\
& \left.\left.+C\left(s, k_{2}\right)|s|^{3} \sum_{l=1}^{n} \chi\left(\left|\phi_{n}\left(X_{l}\right)\right| \geqslant \epsilon b_{n}^{-1}\right)+O\left(n r^{2} b_{n}^{4}\right)\right\}\right] \\
& =n \log \mathbf{E}_{n}\left[\operatorname { e x p } \left\{b_{n} t^{\prime} \vec{f}_{n}\left(X_{1}\right)+\frac{b_{n}^{2}}{2} \phi_{n}^{2}\left(X_{1}\right)\left(1+2 C\left(s, k_{2}\right) \epsilon\right)\right.\right. \\
& \left.\left.+C\left(s, k_{2}\right)|s|^{3} \chi\left(\left|\phi_{n}\left(X_{1}\right)\right| \geqslant \epsilon b_{n}^{-1}\right)+O\left(r^{2} b_{n}^{4}\right)\right\}\right] .
\end{aligned}
$$

Expanding in the Taylor series, we get

$$
\begin{aligned}
\log U_{1 n} \leqslant n \log \mathbf{E}_{n} & {\left[1+b_{n} t^{\prime} \vec{f}_{n}\left(X_{1}\right)+\frac{b_{n}^{2}}{2}\left(t^{\prime} \vec{f}_{n}\left(X_{1}\right)\right)^{2}\right.} \\
& \left.+\frac{b_{n}^{2}}{2} \phi_{n}^{2}\left(X_{1}\right)\left(1+2 C\left(s, k_{2}\right) \epsilon\right)+C\left(s, t, k_{1}, k_{2}\right) \omega_{n}+O\left(r^{2} b_{n}^{4}\right)\right]
\end{aligned}
$$

with

$$
\begin{aligned}
\omega_{n} & =\omega_{1 n}+\omega_{2 n}+\omega_{3 n}+\omega_{4 n}+\omega_{5 n}, \\
\omega_{1 n} & =\frac{b_{n}^{3}}{6}\left|t^{\prime} \vec{f}_{n}\left(X_{1}\right)\right|^{3}, \quad \omega_{2 n}=3 \frac{b_{n}^{3}}{2}\left|t^{\prime} \vec{f}_{n}\left(X_{1}\right)\right| \phi_{n}^{2}\left(X_{1}\right), \quad \omega_{3 n}=\frac{b_{n}^{4}}{8} \phi_{n}^{4}\left(X_{1}\right), \\
\omega_{4 n} & =\frac{b_{n}^{4}}{12}\left(t^{\prime} \vec{f}_{n}\left(X_{1}\right)\right)^{2} \phi_{n}^{2}\left(X_{1}\right), \quad \omega_{5 n}=\chi\left(\left|\phi_{n}\left(X_{1}\right)\right| \geqslant \epsilon b_{n}^{-1}\right) .
\end{aligned}
$$

We have

$$
\begin{aligned}
& \omega_{1 n} \leqslant b_{n}^{3}\left|t^{\prime} \vec{f}_{n}\left(X_{1}\right)\right|^{3} \chi\left(\left|t^{\prime} \vec{f}_{n}\left(X_{1}\right)\right|<\epsilon b_{n}^{-1}\right)+\chi\left(\epsilon b_{n}^{-1}<\left|t^{\prime} \vec{f}_{n}\left(X_{1}\right)\right|<b_{n}^{-1}\right) \\
& \doteq \omega_{1 n 1}+\omega_{1 n 2}, \\
& \omega_{2 n} \leqslant b_{n}^{3}\left|t^{\prime} \vec{f}_{n}\left(X_{1}\right)\right| \phi_{n}^{2}\left(X_{1}\right) \chi\left(\left|t^{\prime} \vec{f}_{n}\left(X_{1}\right)\right|<\epsilon b_{n}^{-1}\right)
\end{aligned}
$$




$$
\begin{aligned}
&+C\left(s, t, k_{1}, k_{2}\right) \chi\left(\epsilon b_{n}^{-1}<\left|t^{\prime} \vec{f}_{n}\left(X_{1}\right)\right|<b_{n}^{-1}\right) \doteq \omega_{2 n 1}+\omega_{2 n 2}, \\
& \omega_{3 n} \leqslant b_{n}^{4} \phi_{n}^{4}\left(X_{1}\right) \chi\left(\phi_{n}\left(X_{1}\right)<\epsilon b_{n}^{-1}\right)+C \chi\left(\epsilon b_{n}^{-1}<\phi_{n}\left(X_{1}\right)<c b_{n}^{-1}\right) \\
& \doteq \omega_{3 n 1}+\omega_{3 n 2}, \\
& \omega_{4 n} \leqslant b_{n}^{4}\left(t^{\prime} \vec{f}_{n}\left(X_{1}\right)\right)^{2} \phi_{n}^{2}\left(X_{1}\right) \chi\left(\left|t^{\prime} \vec{f}_{n}\left(X_{1}\right)\right|<\epsilon b_{n}^{-1}\right) \\
&+c \chi\left(\epsilon b_{n}^{-1}<\left|t^{\prime} \vec{f}_{n}\left(X_{1}\right)\right|<b_{n}^{-1}\right) \\
& \qquad \omega_{4 n 1}+\omega_{4 n 2} .
\end{aligned}
$$

Using (3.1), we get

$$
\begin{array}{cc}
\mathbf{E}_{n}\left[\omega_{1 n 1}\right] \leqslant c \epsilon|t| b_{n}^{2} \mathbf{E}_{n}\left[\left(t^{\prime} \vec{f}_{n}\left(X_{1}\right)\right)^{2}\right], & \mathbf{E}_{n}\left[\omega_{2 n 1}\right] \leqslant c \epsilon|t| b_{n}^{2} \mathbf{E}_{n}\left[\phi_{n}^{2}\left(X_{1}\right)\right], \\
\mathbf{E}_{n}\left[\omega_{3 n 1}\right] \leqslant c \epsilon^{2}|s|^{2} b_{n}^{2} \mathbf{E}_{n}\left[\phi_{n}^{2}\left(X_{1}\right)\right], & \mathbf{E}_{n}\left[\omega_{4 n 1}\right] \leqslant c \epsilon^{2}|t|^{2} b_{n}^{2} \mathbf{E}_{n}\left[\phi_{n}^{2}\left(X_{1}\right)\right]
\end{array}
$$

and

$$
\begin{aligned}
& \mathbf{E}_{n}\left[\omega_{5 n}\right] \leqslant \epsilon^{-2} b_{n}^{2} \mathbf{E}_{n}\left[\phi_{n}^{2}\left(X_{1}\right) \chi\left(\left|\phi_{n}\left(X_{i}\right)\right| \geqslant \epsilon b_{n}^{-1}\right)\right]=o\left(\epsilon^{-2} b_{n}^{2}\right), \\
& \mathbf{E}_{n}\left[\chi\left(\epsilon b_{n}^{-1}<\left|t^{\prime} \vec{f}_{n}\left(X_{1}\right)\right|<b_{n}^{-1}\right)\right] \\
& \leqslant \epsilon^{-2} b_{n}^{2} \mathbf{E}_{n}\left[\left|t^{\prime} \vec{f}_{n}\left(X_{1}\right)\right|^{2} \chi\left(\epsilon b_{n}^{-1}<\left|t^{\prime} \vec{f}_{n}\left(X_{1}\right)\right|\right)\right]=o\left(\epsilon^{-2} b_{n}^{2}\right)
\end{aligned}
$$

where the last inequalities in (6.14) and (6.15) follows from A and (3.5), (3.6).

Hence, we get $\mathbf{E}_{n}\left[\omega_{n}\right]=o\left(b_{n}^{2}\right)$. Therefore we have

$$
\log \left(U_{1 n}\right) \leqslant-\frac{n b_{n}^{2}}{2}\left(2\left\langle t^{\prime} \vec{f}, H\right\rangle-t^{\prime} R_{f} t-s^{\prime} R_{g} s\right)(1+O(1)) \doteq v_{n} .
$$

Implementing the Hoelder's inequality, we get

$$
U_{2 n} \leqslant\left(\mathbf{E}_{n}\left[W_{n}^{1+\delta}\right]\right)^{\frac{1}{1+\delta}}\left(\mathbf{P}\left(\bar{A}_{n}\right)\right)^{\frac{\delta}{1+\delta}} .
$$

Using (6.13), we get

$$
\begin{aligned}
\mathbf{E}_{n}\left[W_{n}^{1+\delta}\right] & \leqslant \mathbf{E}_{n}\left[\operatorname { e x p } \left\{( 1 + \delta ) \left(b_{n} \sum_{i=1}^{n} t^{\prime} \vec{f}_{n}\left(X_{i}\right)\right.\right.\right. \\
& \left.\left.\left.+b_{n}^{2} \sum_{i=1}^{n} \phi_{n}^{2}\left(X_{i}\right)\left(1+2 C\left(s, k_{2}\right) \epsilon\right)+2 C\left(s, k_{2}\right) \sum_{i=1}^{n} \chi\left(\phi_{n}\left(X_{i}\right)>\epsilon b_{n}^{-1}\right)\right)\right\}\right] .
\end{aligned}
$$

Hence, repeating the estimates for $U_{1 n}$, we get

$$
\begin{aligned}
& \mathbf{E}_{n}\left[W_{n}^{1+\delta}\right] \\
& \quad \leqslant \exp \left\{-\frac{(1+\delta) n b_{n}^{2}}{2}\left(2<t^{\prime} \vec{f}, H>-t^{\prime} R_{f} t-s^{\prime} R_{g} s\right)(1+O(1))\right\} .
\end{aligned}
$$

Note that (3.1) and B1 implies (3.4) and (3.4) implies

$$
\lim _{n \rightarrow \infty}\left(n r^{2} b_{n}^{2}\right)^{-1} \log \left(n \mathbf{P}\left(|f(X)|>r n b_{n}\right)\right)=-\infty
$$

for all $r>1$.

Hence, by Theorem 2.4 in [2], we get

$$
\log \mathbf{P}_{n}\left(\bar{A}_{n}\right) \leqslant-c r^{2} n b_{n}^{2} .
$$

By (6.16) -6.18$)$, we get

$$
U_{2 n}=o\left(U_{1 n}\right)
$$

if $r$ is sufficiently large. This completes the proof of upper bound for $I_{n}$. 
The proof of lower bound is based on similar estimates. Denote

$$
\begin{aligned}
& B_{n}=\left\{x_{1}, \ldots, x_{n}:\left|f_{n i}\left(x_{s}\right)\right|<\epsilon b_{n}^{-1},\right.\left|g_{n j}\left(x_{s}\right)\right|<\epsilon b_{n}^{-1}, \\
&\left.1 \leqslant s \leqslant n, 1 \leqslant i \leqslant k_{1}, 1 \leqslant j \leqslant k_{2}\right\} .
\end{aligned}
$$

By (3.1), 6.14) and (6.15), we get

$$
\begin{array}{r}
\mathbf{P}_{n}\left(\left|f_{n i}\left(X_{1}\right)\right|>\epsilon b_{n}^{-1}\right) \\
<\epsilon^{-2} b_{n}^{2} \mathbf{E}_{n}\left[f_{n i}^{2}\left(X_{1}\right) \chi\left(\left|f_{n i}\left(X_{1}\right)\right|>\epsilon b_{n}^{-1}\right)\right]=o\left(\epsilon^{-2} b_{n}^{2}\right) .
\end{array}
$$

Estimating similarly $\mathbf{P}_{n}\left(\left|g_{n i}\left(X_{1}\right)\right|>\epsilon b_{n}^{-1}\right)$, we get

$$
\begin{aligned}
\mathbf{P}\left(B_{n}\right)=\prod_{i=1}^{k_{1}}\left(1-\mathbf{P}\left(\left|f_{n i}\left(X_{1}\right)\right|>\epsilon b_{n}^{-1}\right)\right)^{n} \prod_{i=1}^{k_{2}}\left(1-\mathbf{P}\left(\left|g_{n i}\left(X_{1}\right)\right|>\epsilon b_{n}^{-1}\right)\right)^{n} \\
=\exp \left\{-o\left(n b_{n}^{2}\right)\right\}
\end{aligned}
$$

Hence

$$
\begin{gathered}
I_{n} \geqslant \mathbf{E}_{n}\left[\exp \left\{b_{n} \sum_{i=1}^{n} t^{\prime} \vec{f}_{n}\left(X_{i}\right)\right\}\left(\frac{1}{n} \sum_{i=1}^{n} \exp \left\{b_{n} s^{\prime}\left(\vec{g}_{n}\left(X_{i}\right)-\bar{g}_{n}\right)\right\}\right)^{n} \chi\left(B_{n}\right)\right] \\
=\mathbf{E}_{n}\left[\exp \left\{b_{n} \sum_{i=1}^{n} t^{\prime} \vec{f}_{n}\left(X_{i}\right)\right\}\left(\frac{1}{n} \sum_{i=1}^{n} \exp \left\{b_{n} s^{\prime}\left(\vec{g}_{n}\left(X_{i}\right)-\bar{g}_{n}\right)\right\}\right)^{n} \mid B_{n}\right] \\
\times \exp \left\{-o\left(n b_{n}^{2}\right)\right\} \doteq I_{2 n} \exp \left\{-o\left(n b_{n}^{2}\right)\right\} .
\end{gathered}
$$

Expanding in the Taylor series, we get

$$
\begin{aligned}
I_{2 n} & \geqslant \mathbf{E}_{n}\left[\operatorname { e x p } \{ b _ { n } \sum _ { i = 1 } ^ { n } t ^ { \prime } \vec { f } _ { n } ( X _ { i } ) \} \left(1+\frac{b_{n}^{2}}{2 n} \sum_{i=1}^{n}\left(s^{\prime}\left(\vec{g}_{n}\left(X_{i}\right)-\bar{g}_{n}\right)\right)^{2}\right.\right. \\
& \left.\left.-C\left(s, k_{2}\right) \frac{b_{n}^{3}}{n} \sum_{i=1}^{n}\left|s^{\prime}\left(\vec{g}_{n}\left(X_{i}\right)-\bar{g}_{n}\right)\right|^{3}\right)^{n} \mid B_{n}\right] \geqslant \\
\geqslant & \mathbf{E}_{n}\left[\exp \left\{b_{n} \sum_{i=1}^{n} t^{\prime} \vec{f}_{n}\left(X_{i}\right)\right\}\right. \\
& \left.\times\left(1+\frac{b_{n}^{2}}{2 n}(1-2 \epsilon) \sum_{i=1}^{n}\left(s^{\prime}\left(\vec{g}_{n}\left(X_{i}\right)-\bar{g}_{n}\right)\right)^{2}\right)^{n} \mid B_{n}\right] \doteq I_{3 n}
\end{aligned}
$$

where the last inequality follows from

$$
\sum_{i=1}^{n}\left|s^{\prime}\left(\vec{g}_{n}\left(X_{i}\right)-\bar{g}_{n}\right)\right|^{3} \leqslant 2 \epsilon b_{n}^{-1} \sum_{i=1}^{n}\left(s^{\prime}\left(\vec{g}_{n}\left(X_{i}\right)-\bar{g}_{n}\right)\right)^{2} .
$$

Since $\log (1+x) \geqslant 1+x-x^{2}$ for $x>0$, then 


$$
\begin{aligned}
I_{3 n} & =\mathbf{E}_{n}\left[\exp \left\{b_{n} \sum_{i=1}^{n} t^{\prime} \vec{f}_{n}\left(X_{i}\right)\right\}\right. \\
& \left.\times \exp \left\{n \ln \left(1+\frac{b_{n}^{2}}{2}(1-2 \epsilon) \sum_{i=1}^{n}\left(s^{\prime}\left(\vec{g}_{n}\left(X_{i}\right)-\bar{g}_{n}\right)\right)^{2}\right)\right\} \mid B_{n}\right] \\
\geqslant & \mathbf{E}_{n}\left[\operatorname { e x p } \left\{b_{n} \sum_{i=1}^{n} t^{\prime} \vec{f}_{n}\left(X_{i}\right)+\frac{b_{n}^{2}}{2}(1-2 \epsilon) \sum_{i=1}^{n}\left(s^{\prime}\left(\vec{g}_{n}\left(X_{i}\right)-\bar{g}_{n}\right)\right)^{2}\right.\right. \\
& \left.\left.-\frac{b_{n}^{4}}{4 n}\left(\sum_{i=1}^{n}\left(s^{\prime}\left(\vec{g}_{n}\left(X_{i}\right)-\bar{g}_{n}\right)\right)^{2}\right)^{2}\right\} \mid B_{n}\right] \\
\geqslant & \mathbf{E}_{n}\left[\operatorname { e x p } \left\{b_{n} \sum_{i=1}^{n} t^{\prime} \vec{f}_{n}\left(X_{i}\right)\right.\right. \\
& \left.\left.+\frac{b_{n}^{2}}{2}\left(1-2 \epsilon-4 \epsilon^{2}\right) \sum_{i=1}^{n}\left(s^{\prime}\left(\vec{g}_{n}\left(X_{i}\right)-\bar{g}_{n}\right)\right)^{2}\right\} \mid B_{n}\right] \doteq I_{4 n}
\end{aligned}
$$

where the last inequality follows from

$$
\frac{b_{n}^{4}}{4 n}\left(\sum_{i=1}^{n}\left(s^{\prime}\left(\vec{g}_{n}\left(X_{i}\right)-\bar{g}_{n}\right)\right)^{2}\right)^{2} \leqslant \epsilon^{2} b_{n}^{2} \sum_{i=1}^{n}\left(s^{\prime}\left(\vec{g}_{n}\left(X_{i}\right)-\bar{g}_{n}\right)\right)^{2} .
$$

Estimating similarly to the proof of upper bound, we get

$$
\begin{aligned}
& \left(n b_{n}^{2}\right)^{-1} \ln I_{4 n} \\
& \quad=-\frac{n b_{n}^{2}}{2}\left(-2<t^{\prime} \vec{f}, H>-t^{\prime} R_{f} t-\left(1-2 \epsilon-2 \epsilon^{2}\right) s^{\prime} R_{g} s\right)(1+O(1)) .
\end{aligned}
$$

Since the choice of $\epsilon>0$ is arbitrary, this completes the proofs of lower bound and Lemma 6.5

\section{Proof of Theorem 3.2}

It suffices to show that

$$
-\log \mathbf{P}\left(\sum_{i=1}^{n} Y_{i}^{*}>n e_{n}\right)=o\left(n e_{n}^{2}\right) .
$$

Define the events $A_{n i}=U_{n i} \cup V_{n i}, 1 \leqslant i \leqslant n$, where

$$
U_{n i}=\left\{Y_{i}:\left|Y_{i}\right|<b_{n}^{-1}\right\} \text { and } V_{n i}=\left\{Y_{i}: r_{n}<Y_{i}\right\} \text {. }
$$

Denote $A_{n}=\bigcap_{i=1}^{n} A_{n i}$. Using (3.1), we get

$$
\mathbf{P}\left(A_{n}\right)>1-\mathbf{P}\left(\max _{1 \leqslant i \leqslant n}\left|Y_{i}\right|>b_{n}^{-1}\right)>1-n \mathbf{P}\left(\left|Y_{1}\right|>b_{n}^{-1}\right)=1+o(1) .
$$

Denote $\mathbf{P}_{c n}$ the conditional probability measure of $Y_{1}$ given $Y_{1} \in A_{n 1}$.

Using (7.2), we get

$$
\begin{aligned}
\mathbf{P}\left(\sum_{i=1}^{n} Y_{i}^{*}>n e_{n}\right) & \geqslant \mathbf{P}\left(\sum_{i=1}^{n} Y_{i}^{*}>n e_{n} \mid A_{n}\right) \mathbf{P}\left(A_{n}\right) \\
& =\mathbf{P}_{c n}\left(\sum_{i=1}^{n} Y_{i}^{*}>n e_{n}\right)(1+o(1)) .
\end{aligned}
$$


Thus, it suffices to prove (7.1) for the probability measures $\mathbf{P}_{c n}$ instead of $\mathbf{P}$. Denote $p_{n}=\mathbf{P}_{c n}\left(Y_{1}>r_{n}\right)$. By (3.1), we get $n p_{n} \rightarrow 0$ as $n \rightarrow \infty$. Define the events

$W_{n}\left(k_{n}\right)=\left\{Y_{1}, \ldots, Y_{n}: n-k_{n}\right.$ random variables $Y_{1}, \ldots, Y_{n}$ belong $\left(0, b_{n}^{-1}\right)$ and $k_{n}$ random variables $Y_{1}, \ldots, Y_{n}$ belong $\left.\left(r_{n}, \infty\right)\right\}$.

Suppose that $k=k_{n} \rightarrow \infty$ as $n \rightarrow \infty$ and

$$
\lim _{n \rightarrow \infty} k_{n} n p_{n}=0, \quad \lim _{n \rightarrow \infty}\left(r_{n} e_{n}\right)^{-1} \log \frac{n e_{n}}{r_{n} k_{n}}=0 .
$$

Implementing the Stirling formula, we get

$$
\begin{aligned}
v_{n} & \doteq \mathbf{P}_{c n}\left(W_{n}(k)\right)=\frac{n !}{(n-k) ! k !} p_{n}^{k}\left(1-p_{n}\right)^{n-k} \\
= & (2 \pi)^{-1 / 2} \exp \{(n+1 / 2) \log n-(n-k+1 / 2) \log (n-k) \\
& \left.-(k+1 / 2) \log k+k \log p_{n}+(n-k) \log \left(1-p_{n}\right)\right\}(1+o(1)) \\
= & \exp \left\{-(n-k+1 / 2) \log \frac{n-k}{n\left(1-p_{n}\right)}-k \log \frac{k}{n p_{n}}(1+o(1))\right\} \\
= & \exp \left\{-n(1-k / n)\left(-k / n+p_{n}\right)(1+o(1))-k \log \left[k /\left(n p_{n}\right)\right](1+o(1))\right\} \\
= & \exp \left\{\left(k-n p_{n}-k \log \left(k /\left(n p_{n}\right)\right)(1+o(1))\right\}\right. \\
= & \exp \left\{-k \log \frac{k}{n p_{n}}(1+o(1))\right\} .
\end{aligned}
$$

It follows from (3.2) and (7.4) that we can choose $k=k_{n}$ such that

$$
\left|\log v_{n}\right|=O\left(k_{n}\left|\log \left(n p_{n}\right)\right|\right)=o\left(n e_{n}^{2}\right) .
$$

Define the random variable $l_{n}$ which equals the number of $Y_{i}^{*}, 1 \leqslant i \leqslant n$ such that $Y_{i}^{*} \in\left(r_{n}, \infty\right)$. Denote $u_{n}=c \frac{n e_{n}}{r_{n}}=c \frac{n e_{n}^{2}}{r_{n} e_{n}} c>1$ and put $m_{n}=\left[u_{n}\right]$. Suppose that $\frac{u_{n}}{k_{n}} \rightarrow \infty$ as $n \rightarrow \infty$. Then, estimating similarly to (7.4), we get

$$
\mathbf{P}_{c}\left(l_{n}>u_{n} \mid W_{n}\left(k_{n}\right)\right)=\exp \left\{-u_{n} \log \frac{u_{n}}{k_{n}}(1+o(1))\right\} .
$$

Denote $c_{1}=c-1$. Denote $Y^{1 *} \leqslant \ldots \leqslant Y^{n *}$ the order statistis of $Y_{1}^{*}, \ldots, Y_{n}^{*}$.

The event $\left\{Y_{1}^{*}, \ldots, Y_{n}^{*}: \sum_{i=1}^{n} Y_{i}^{*}>n e_{n}\right\}$ contains the event

$$
\begin{gathered}
U_{n}=\left\{Y_{1}^{*}, \ldots, Y_{n}^{*}: \sum_{j=1}^{n-m_{n}} Y^{j *}>-c_{1} n e_{n},\left|Y^{j *}\right|<b_{n}^{-1},\right. \\
\left.1 \leqslant j \leqslant n-m_{n}, Y^{t *}>r_{n}, n-m_{n}<t \leqslant n\right\},
\end{gathered}
$$

since, if $U_{n}$ holds, then we have

$$
\sum_{t=n-m_{n}-1}^{n} Y^{t *}>r_{n} m_{n}=c r_{n} \frac{n e_{n}}{r_{n}}=c n e_{n} .
$$

Thus it suffices to prove that

$$
\log \mathbf{P}_{c}\left(U_{n}\right)=o\left(n e_{n}^{2}\right) .
$$

We have

$$
\begin{aligned}
& \mathbf{P}_{c}\left(U_{n}\right) \geqslant \mathbf{P}_{c}\left(l_{n}=m_{n}\right) \mathbf{P}_{c}\left(\sum_{i=1}^{n-m_{n}} Y_{i}^{*}>-c_{1} n e_{n},\left|Y_{i}^{*}\right|<b_{n}^{-1}, 1 \leqslant i \leqslant n-m_{n}\right) \\
& \geqslant \mathbf{P}_{c}\left(l_{n}=m_{n} \mid W_{n}\left(k_{n}\right)\right) \\
& \quad \times \mathbf{P}_{c}\left(W_{n}\left(k_{n}\right)\right) \mathbf{P}_{c}\left(\sum_{i=1}^{n-m_{n}} Y_{i}^{*}>-c_{1} n e_{n},\left|Y_{i}^{*}\right|<b_{n}^{-1}, 1 \leqslant i \leqslant n-m_{n}\right) .
\end{aligned}
$$


Denote $q_{n}=\mathbf{P}_{c}\left(\left|Y_{1}\right|<b_{n}^{-1}\right)$. Define the conditional probability measure $\mathbf{P}_{b_{n}}$ of random variable $Y_{1}$ given $\left|Y_{1}\right|<b_{n}^{-1}$.

We have

$$
\begin{aligned}
\mathbf{P}_{c}\left(\left|Y_{1}^{*}\right|<b_{n}^{-1}\right) & =\sum_{i=1}^{n} \frac{n !}{(n-i) ! i !} q_{n}^{i}\left(1-q_{n}\right)^{n-i} \frac{i}{n} \\
& =q_{n} \sum_{i=1}^{n} \frac{(n-1) !}{(n-i) !(i-1) !} q_{n}^{i-1}\left(1-q_{n}\right)^{n-i}=q_{n} .
\end{aligned}
$$

We have

$$
\begin{aligned}
& \mathbf{P}_{c}\left(\sum_{i=1}^{n-m_{n}} Y_{i}^{*}>-c_{1} n e_{n}|| Y_{i}^{*} \mid<b_{n}^{-1}, 1 \leqslant i \leqslant n-m_{n}\right) \\
& \quad=1-\mathbf{P}_{c}\left(\sum_{i=1}^{n-m_{n}} Y_{i}^{*}<-c_{1} n e_{n}|| Y_{i}^{*} \mid<b_{n}^{-1}, 1 \leqslant i \leqslant n-m_{n}\right) .
\end{aligned}
$$

By Chebyshev inequality, using (7.8), we get

$$
\begin{aligned}
\mathbf{P}_{c}\left(\sum_{i=1}^{n-m_{n}} Y_{i}^{*}<-c_{1} n e_{n}|| Y_{i}^{*} \mid<b_{n}^{-1}, 1 \leqslant i \leqslant n-m_{n}\right) \\
\leqslant \frac{n-m_{n}}{c_{1}^{2}\left(n-m_{n}\right)^{2} e_{n}^{2}} \mathbf{E}_{c}\left[\operatorname{Var}_{\widehat{\mathbf{P}}_{n}}\left(Y_{1}^{*}|| Y_{1}^{*} \mid<b_{n}^{-1}\right)\right] \\
=\frac{q_{n}^{2}}{c_{1}^{2}\left(n-m_{n}\right) e_{n}^{2}} \sum_{t=0}^{n} C_{n}^{t} q_{n}^{t}\left(1-q_{n}\right)^{n-t} \mathbf{E}_{b_{n}} \\
\times\left[(n-t)^{-1} \sum_{i=1}^{n-t}\left(Y_{i}-(n-t)^{-1} \sum_{j=1}^{n-t} Y_{j}\right)^{2}\right] \\
=\frac{q_{n}^{2}}{c_{1}^{2}\left(n-m_{n}\right) e_{n}^{2}} \sum_{t=0}^{n} C_{n}^{t} q_{n}^{t}\left(1-q_{n}\right)^{n-t} \frac{t-1}{t} \operatorname{Var}_{b_{n}}[Y] \\
\leqslant \frac{q_{n}^{2}}{c_{1}^{2}\left(n-m_{n}\right) e_{n}^{2}} \operatorname{Var}_{b_{n}}[Y]
\end{aligned}
$$

and

$$
\lim _{n \rightarrow \infty} q_{n}^{2} \operatorname{Var}_{b_{n}}[Y]=\operatorname{Var}[Y]
$$

Using (7.4) and (7.6), we get

$$
\begin{aligned}
& \mathbf{P}_{c}\left(l_{n}=m_{n} \mid W_{n}\left(k_{n}\right)\right) \mathbf{P}_{c}\left(W_{n}\left(k_{n}\right)\right) \\
& \quad=\exp \left\{-\frac{c n e_{n}^{2}}{r_{n} e_{n}} \log \frac{n e_{n}}{r_{n} k_{n}}-c k_{n} \log \frac{k_{n}}{n p_{n}}(1+o(1))\right\} \\
& \quad=\exp \left\{-o\left(n e_{n}^{2}\right)\right\}
\end{aligned}
$$

where the last inequality follows from (77.3), (7.5). Now (7.7) follows from (7.9) 7.12$)$. This completes the proof of Theorem 3.2 .

\section{REFERENCES}

[1] A. K. Aleskevičiene, Large and moderate deviations for L-statistics. — Lithuanian Math. J. 31 (1991), 145-156.

[2] M. A. Arcones, Moderate deviations of empirical processes. - In: Stochastic Inequalities and Applications, E. Giné, C. Houdré, and D. Nualart (eds.) Birkhäuser Boston (2003), pp. $189-212$.

[3] M. A. Arcones, Large deviations for M-estimators. - Ann. Inst. Math. Statist. 58 (2006), $21-52$. 
[4] A.A. Borovkov and A.A. Mogulskii. On probabilities of large deviations in topological spaces. II. - Siberian Mathematical Journal, 21(5), (1980), 12-26.

[5] E. Bolthausen, On the probability of large deviations in Banach spaces. - Ann. Probab 12 (1984), 427-435.

[6] N. R. Chaganty, R. L. Karandikar, Some properties of the Kullback-Leibler number. Sankhyā, A, 58 (1996), 69-80.

[7] N. R. Chaganty, Large deviations for joint distributions and statistical applications. Sankhya Ā, 59 (1997), 147-166.

[8] A. de Acosta, On large deviations of empirical measures in the $\tau$-topology. - J. Appl. Probab. 31A (1994), 41-47.

[9] R. Dasgupta, Bootstrap of deviation probabilities with applications. - J. Multivariate Anal. 101 (2010), 2137-2148.

[10] A. Dembo, O. Zeitouni. Large Deviations Techniques and Applications. Jones and Bartlett, Boston, 1993

[11] M. D. Donsker, S. R. S. Varadhan, Asymptotic evaluation of certain Markov process expectations for large time III, Comm. Pure Appl. Math. 29 (1976), 389-461.

[12] B. Efron, Bootstrap methods: another look at the jackknife. - Ann. Stat. 7 (1979), 1-26.

[13] P. Eichelsbacher, U. Schmock, Large deviations of U-empirical measures in strong topologies and applications. - Ann. Inst. Henri Poincaré. Probab. Statist. 38 (2002), 779-797.

[14] P. Eichelsbacher, M. Löwe. Moderate deviations for i.i.d. random variables. - ESAIM: Probab. Statist. 7 (2003), 207-216.

[15] M. S. Ermakov, Importance sampling for simulation of moderate deviation probabilities of statistics. - Statist. Decision, 25 (2007), 265-284.

[16] F. Gao, X. Zhao, Delta method in large deviations and moderate deviations for estimators. - Ann. Statist. 39 (2011), 1211-1240

[17] P. Groeneboom, J. Oosterhoff, F. H. Ruymgaart, Large deviation theorems for empirical probability measures. — Ann. Probab. 7 (1979), 553-586.

[18] P. Hall, On the relative performance of bootstrap and Edgeworth approximations of a distribution function. - J. Multivariate Anal. 35, (1990), 108-129.

[19] T. Inglot, W. C. M. Kallenberg, T. Ledwina. Strong moderate deviation theorems. - Ann. Probab. 20 (1992), 987-1003.

[20] J. Jureckova, W. C. M. Kallenberg, N. Veraverbeke, Moderate and Cramer type large deviation theorems for M-estimators. - Statist. Probab. Lett. 6 (1988), 191-199.

[21] C. Leonard, J. Najim, An extension of Sanov's theorem. Application to the Gibbs conditioning principle. - Bernoulli 8, (2002), 721-743.

[22] D. Li, A. Rosalski, D. K. Al-Mutairi, A large deviation principle for bootstrapped sample means. - Proc. Amer. Math. Soc. 130 (2001), 2133-2138.

[23] V. V. Petrov, Sums of Independent Random Variables. Springer, New York, 1975.

[24] I. Sanov. On the probabilities of large deviations of random variables. Mathematicheskii Sbornik, 42, (1957), 70-95 (In Russian) English translation:Selected Translations in Mathematical Statistics and Probability, 1: 213-244, 1961.

[25] R. J. Serfling, Approximation Theorems of Mathematical Statistics. Wiley, New York, 1980.

[26] L. Saulis and V.Statulevichius. Limit Theorems for Large Deviations. Mokslas Publishers. Vilnius, 1989.

[27] A. W. van der Vaart, J. A. Wellner, Weak Convergence and Empirical Processes with Applications to Statistics. Springer, New York, 1996.

[28] A. Wood, Bootstrap relative errors and subexponential destributions. - Bernoulli 6 (2000), 809-834.

Ermakov M. S. Large Deviation Principle for moderate deviation probabilities of empirical bootstrap measure.

We prove two Large deviations principles (LDP) in the zone of moderate deviation probabilities. First we establish LDP for the conditional distributions of moderate deviations of empirical bootstrap measures given empirical probability measures. Second we establish LDP for the joint distributions of empirical measure and bootstrap empirical measures. Using these LDPs, similar LDPs for statistical differentiable functionals can be established. The LDPs for moderate deviations of 
empirical quantile processes and empirical bootstrap copula function are provided as illustration of these results.

Mechanical Engineering Problems Institute RASc, Bolshoy Pr. V.O., 61, 199178 St.Petersburg, Russia, Saint-Petersburg State University, University pr. 28, Petrodvoretz, 198504, St.-Petersburg, Russia

E-mail address: erm2512@mail.ru 